\title{
COMPARISON OF MATRIX CONVERTER STABILIZATION TECHNIQUES BASED ON THE DAMPING RESISTOR AND DIGITAL FILTER APPROACHES FOR BIDIRECTIONAL POWER FLOW CONTROL \\ ISSN 1751-8644 doi: 0000000000 www.ietdl.org
}

\author{
Zahra Malekjamshidi'*, Mohammad Jafari' ${ }^{1}$, Jianguo Zhu ${ }^{2}$, Dan Xiao ${ }^{3}$ \\ ${ }^{1}$ School of Electrical and Data Engineering, University of Technology Sydney, NSW, 2007, Sydney, Australia \\ ${ }^{2}$ School of Electrical and Information Engineering, The University of Sydney, NSW, 2006, Sydney, Australia \\ ${ }^{3}$ School of Electrical Engineering and Telecommunications, University of New South Wales, NSW, 2052, Sydney, Australia \\ ${ }^{*}$ E-mail: z_malekjamshidi@ieee.org
}

\begin{abstract}
:
The matrix converter is becoming a mature technology with its specific advantages and limitations, and can be effectively used as an interface link in the future smart grids. In this context, the stability of the converter under different power flow conditions is highly important and needs more clarification. The input inductor-capacitor filter can significantly impact the stability of the converter when the output is tightly regulated, especially in bidirectional power flow control applications where a low-impedance source is connected to the converter output. This paper investigates the matrix converter stability for bidirectional power flow control, considering the input filter and other parameters of the system. A detailed analysis of two commonly used active and passive stabilization techniques known as digital filter and damping resistor approaches is presented, and a third method based on a combination of these two methods is suggested in this paper. The converter stability region for the proposed technique is determined by using the small-signal model of the converter. The converter performance for the methods is compared in terms of the efficiency, stability, transients and quality of the input and output currents. Numerical simulations and experimental tests are conducted on a prototype direct matrix converter to validate the proposed method.
\end{abstract}

\section{Introduction}

Matrix converters (MCs) have been attractive for research over the last decades due to their interesting features, such as direct frequency conversion, high power density and bidirectional power flow $[1,2]$. They can be used as a grid-connected converter to control the power flow and convert it into a suitable AC form due to their abilities such as bidirectional power flow control, controllable input power factor and sinusoidal input and output currents. MCs are particularly attractive for variable frequency wind and marine turbine generators and high-speed turbine generators [3-5].

In general, a small LC low-pass filter is used at the input side of the converter to eliminate the input current spikes. Considering optimal cost and size, a simple LC filter can adequately attenuate the high order harmonics to an acceptable level. As the harmonic contents of the input current are introduced at the integer multiples of the switching frequency, it is possible to smooth the supply current, by proper selection of the input filter parameters.

When the output variables of the power electronic converters are tightly regulated, the converter behaves like a constant power load (CPL) which presents a negative incremental input resistance. The negative incremental resistance of the CPLs can destabilize the input LC filter and the system [6, 7] depending on the system parameters. The instability related to the negative input resistance depends on the system parameters and can be improved by changing the input filter parameters, however, this is often undesirable and not recommended. Furthermore, the input filter should be designed to satisfy the EMC requirements and provide attenuation at the switching frequency considering the limits on the total harmonic distortion (THD) of the input source current. As the MC is known as an all-silicon converter and does not require large energy storage components, the size of the input filter components should be minimized, and the input

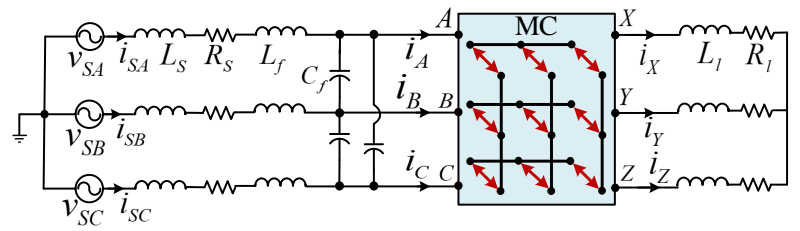

Fig. 1: Schematic of the DMC with LC input filter and RL load

displacement factor is set at unity. Therefore, the resultant instability needs to be resolved by different logical methods.

In this regard, a large amount of research has been done on destabilizing effects of CPLs in multi-converter power electronic systems, DC-DC and AC-DC converters, and different strategies have been proposed to overcome the problem [6-8]. However, the stability analysis of MCs has not been widely studied, and more research work needs to be done in this field. There are different methods for stabilizing the converters. Adding a damping resistor in parallel with the filter inductor is a basic approach for MC stabilization $[9,10]$. As the inherent damping factor of the LC filter is negligible, adding the damping resistors can reduce the input current oscillation. However, the main drawback of this method is a large amount of power dissipation. Another solution is employing Loss-free resistors using extra power electronic elements like inductors and switches which is complicated and needs extra components [11, 12]. Active techniques are another alternative for stabilization without adding extra elements. The active methods are implemented by modifying the controller using a negative input resistance compensator or applying additional algorithms [8, 13-17]. In this strategy, the input impedance of the 
converter is modified by applying a correction term in the feedback loop. In [17], the stability is achieved by constructing different correction terms for modifying the input admittance of the converter, using additional algorithms in each direction of power flow. Also, the measured input voltage can be filtered out using a digital lowpass filter before applying to the modulation section. In this case, the digital filter can be applied for filtering the amplitude of the input voltages [13-15] or the angle of the input voltage vector $[15,16]$. Besides the above-mentioned issues, there are some practical issues that affect the converter stability. In practical systems, the digital implementation of the modulation and control using digital processors can influence the system stability. This is because of the analog to digital converter and the switching period delay introduced by the control algorithm $[13,16]$. Furthermore, the switching and conduction losses of the converter can improve the system stability as illustrated by a simplified model of the MC in [13]

As the main contribution, this paper presents a comprehensive and analytical comparison of the digital low-pass filter method as an active method, and the damping resistor method for MC stabilization in case of the bidirectional power flow control. The main application is in power flow control between the utility grid and a micro-grid where the system is more prone to instability due to the small output resistance. To resolve the issue, a combination of these two methods is suggested in this paper. A small signal model of the converter is derived for each method, and a detailed stability analysis of the converter using the three techniques is provided. The effect of system parameters on the stability region and converter gain is briefly discussed. The performance of the methods in terms of their efficiency, dynamic response and resultant overshoot in transients, and the quality of the input source current are compared. The stability methods are simulated numerically and verified by the experimental test results on a direct matrix converter (DMC) prototype.

\section{Input Filter Design}

The input filter parameters have considerable effect on the system stability. To design the input filter, THD of the input source current, input power factor, and the filter size and cost should be considered. The input filter design of the MC has been discussed in different literature [18-20].

As the unity input power factor is adjusted for the full load, the minimum power factor $\left(\cos \left(\varphi_{i, \max }\right)\right)$ should not be less than a specific limit for the minimum output power $P_{o, \min }$. In this paper, the minimum input power factor 0.9 in $10 \%$ rated input active power $P_{i, n}$ is considered. The maximum input filter capacitance can be defined as [18]:

$$
\begin{aligned}
& C_{f} \leq \frac{P_{o, \min } \tan \left(\varphi_{i, \max }\right)}{3 \omega_{i} U_{i, n}^{2}} \\
& \cos \left(\varphi_{i, \max }\right)=0.9 \\
& P_{o, \min }=0.1 P_{i, n} \\
& P_{i, n}=3 U_{i, n} I_{i, n}
\end{aligned}
$$

where $U_{i, n}$ and $I_{i, n}$ are the rated input phase voltage and current of the MC, and $\omega_{i}$ is the supply angular frequency. In case of the $7.5 \mathrm{~kW}$ prototype $\mathrm{MC}$ with the nominal input phase voltage of $240 \mathrm{~V} / 50 \mathrm{~Hz}$, the maximum input filter capacitance is $6.7 \mu \mathrm{F}$. For the MC prototype, $C_{f}=6.6 \mu F(3 \times 2.2 \mu F$ film capacitor $)$ [21]. Then, the filter inductance $L_{f}$ can be selected by using the cut-off frequency $f_{c}=\frac{1}{2 \pi \sqrt{L_{f} C_{f}}}$, considering that the resonant frequency is more than twenty times the input frequency $f_{i}$ and less than onethird of the switching frequency $f_{s}$ [22]. For gaining the voltage transfer ratio, the voltage drop across the input filter inductance at the rated input current should be minimized. Selecting $L_{f}=3 \mathrm{mH}$ leads to a cut-off frequency about $1130 \mathrm{~Hz}$. Higher switching frequencies of the converter cause the filter size reduction in the cost of increasing the power loss.

\section{Analysis Strategy of the Stability Methods}

A DMC including an input passive low-pass filter and a three-phase RL load as presented in Fig. 1 is used as the analysis and test platform. The instantaneous voltages across the input of the converter are measured for calculating the duty cycles of the switching sequences. Since the discontinuous switching behavior of power electronic converters causes the stability analysis to be complicated, the modeling approaches of the system like small-signal modeling using the statespace averaging method can approximate the system operation. In this paper, the state-space averaging method is utilized to find the mathematical model of the converter in the form of a state matrix. The stability region of the converter then is determined numerically, using the dominant eigenvalue of the resultant state matrix that is the closest eigenvalue to the imaginary axis $[9,14,15]$. The analysis is performed for the whole range of the voltage gain $q$ to determine the gain limit for the stability region of the converter. The system analysis is performed numerically considering the system parameters as $C_{f}=6.6 \mu F, L_{f}=3 m H, R_{s}=0.5 \Omega$ and $L_{s}=0.4 m H$. According to Fig. $1, C_{f}$ and $L_{f}$ are the input filter parameters, and $R_{s}$ and $L_{s}$ are the line impedance elements. The output load elements $R_{l}$ and $L_{l}$ are selected as $10 \Omega$ and $6 \mathrm{mH}$ respectively. The input and output frequencies are $f_{i}=50 \mathrm{~Hz}$ and $f_{o}=70 \mathrm{~Hz}$. In the following sections, the effectiveness of the widely used stabilization techniques including the damping resistor and digital filter methods are presented in details. The proposed combination technique is also studied as the preferred method to provide a context for comparing their performances.

\section{$4 \quad$ The System Stability Without Applying the Stabilization Strategies}

The linearized state-space equations of the converter which are required for stability analysis can be presented in the matrix form referring to the circuit presented in Fig. 1 as:

$$
\begin{aligned}
\frac{d \Delta X}{d t}=A \Delta X & \\
X= & {\left[\begin{array}{cccccc}
i_{s i(d)} & i_{s i(q)} & v_{i(d)} & v_{i(q)} & i_{o(d)} & i_{o(q)}
\end{array}\right]^{T} } \\
A & =\left[\begin{array}{cccccc}
-\frac{R_{s}}{L_{T}} & \omega_{i} & \frac{-1}{L_{T}} & 0 & 0 & 0 \\
-\omega_{i} & -\frac{R_{s}}{L_{T}} & 0 & \frac{-1}{L_{T}} & 0 & 0 \\
\frac{1}{C_{f}} & 0 & k_{1} & \omega_{i} & -\frac{q}{C_{f}} & 0 \\
0 & \frac{1}{C_{f}} & -\omega_{i} & -k_{1} & 0 & 0 \\
0 & 0 & 0 & 0 & -\frac{R_{l}}{L_{l}} & \omega_{o} \\
0 & 0 & 0 & 0 & -\omega_{o} & -\frac{R_{l}}{L_{l}}
\end{array}\right] \\
k_{1} & =\frac{q^{2} R_{l}}{C_{f}\left|\vec{Z}_{l}\right|^{2}}
\end{aligned}
$$

where $\vec{Z}_{l}=R_{l}+j \omega_{o} L_{l}$ represents the load impedance, and $L_{T}=$ $L_{s}+L_{f}$. Also, $X$ includes the d-q components of the input source current space vector $\vec{i}_{s i}$, input voltage space vector $\vec{v}_{i}$ and the output current space vector $\vec{i}_{o}$, as will be explained in section 7 and Fig. 8 in more details. The system stability limits can be found by numerical analysis of the eigenvalues of the state matrix $A$. In fact, the eigenvalues are the poles of the transfer function [23], and determine the maximum voltage gain limit for stable operation of the $\mathrm{MC}$ as a function of the system parameters. Figs. 2 and 3 illustrate the effects of the system parameters on the converter stability. The presented graphs determine the margin between stable and unstable regions. Figs. $2 \mathrm{a}$ and $2 \mathrm{~b}$ show the positive effects of increasing the filter capacitance $C_{f}$ and decreasing the filter inductance $L_{f}$. As can be seen, without applying any stabilization strategy, the system remains 

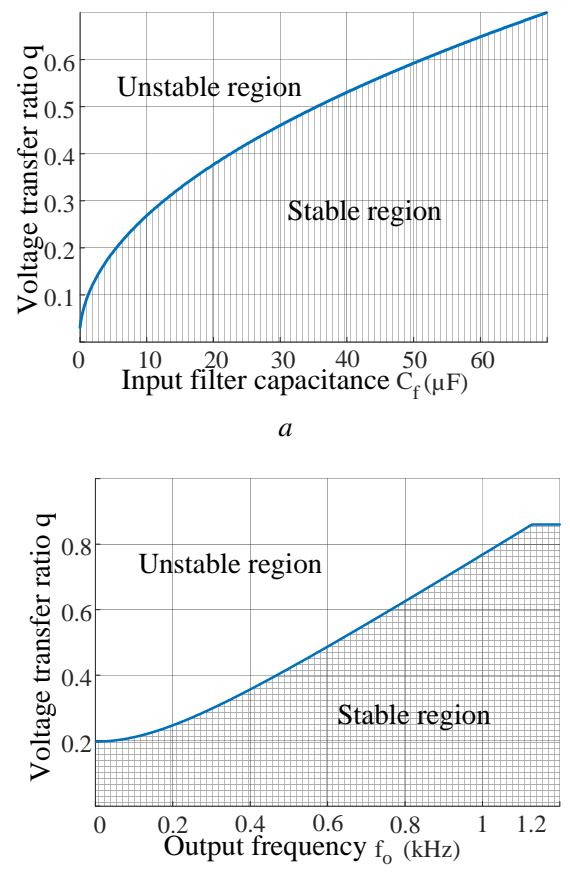

$d$

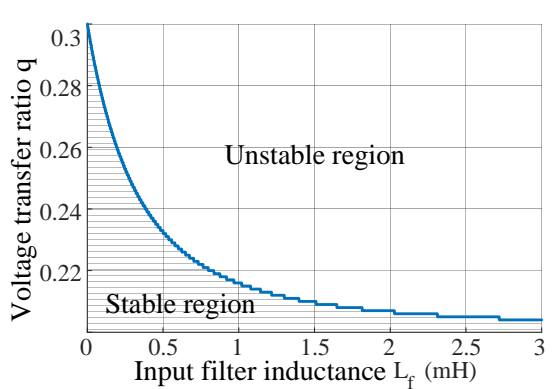

$b$

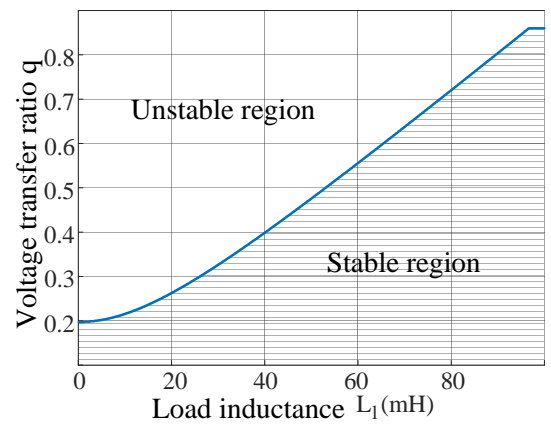

$e$
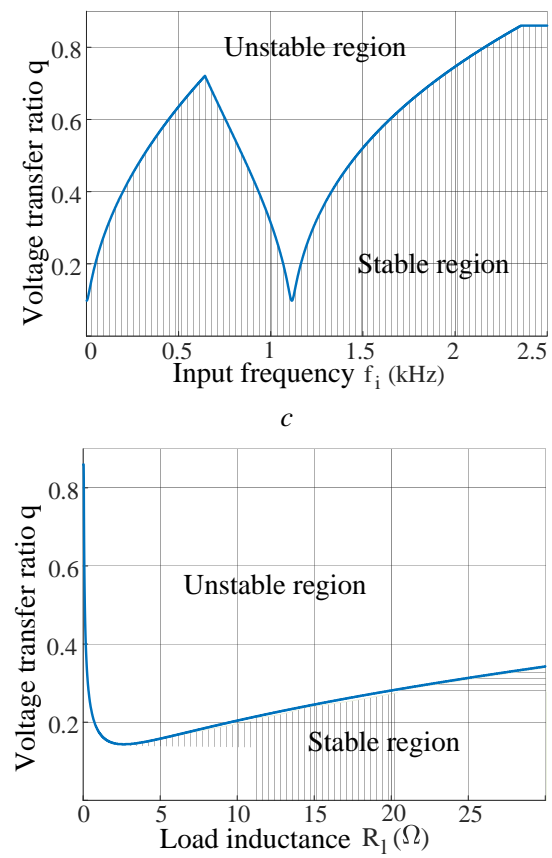

$f$

Fig. 2: The stable region of the system without applying any stabilization strategy, voltage gain $q$ against, (a) Input filter capacitance $C_{f},(b)$ Input filter inductance $L_{f},(c)$ Input frequency $f_{i},(d)$ Output frequency $f_{o},(e)$ Load inductance $L_{l},(f)$ Load resistance $R_{l}$

stable for larger voltage gains by increasing $C_{f}$ or decreasing $L_{f}$, although, the effect of increasing $C_{f}$ is more considerable. On the other hand, the filter parameters should be selected so as to cause minimum displacement angle between the voltage and current of the input source as explained in section 2. Therefore, it is not possible to change these parameters in a wide range, and a suitable stabilization strategy should be applied to increase the stability limit. As demonstrated in Figs. $2 \mathrm{c}$ and $2 \mathrm{~d}$, higher input and output frequencies $f_{i}$ and $f_{o}$ permit a larger voltage gain $q$ in the stability region. In the case of $f_{i}$, for a certain frequency equal to the resonant frequency of the LC filter (including the line inductance), the voltage gain limit reaches to the minimum. Figs. $2 \mathrm{e}$ and $2 \mathrm{f}$ illustrate the effects of the load components $L_{l}$ and $R_{l}$ on the stability. As can be seen, it is possible to expand the stability area by increasing the inductance or the resistance of the output load. In the case of $R_{l}$, at first, by increasing the resistance the voltage gain limit reduces until a specific value of $R_{l}$, and after that, it starts to increase. Also, increasing the line resistance $R_{s}$ as a series damping resistor increases the stability limit as shown in Fig. 3. As can be seen, depending on the other system parameters even increasing the series damping resistor may not lead to the maximum gain, and the voltage gain limit starts to reduce after a special resistance. On the other hand, adding a resistance in series with $L_{f}$ causes efficiency reduction and loss increment. Instead of increasing $R_{s}$, a parallel damping resistor is added to the input filter inductance as will be explained in the next section.

\section{Stabilization Method Using Parallel Damping Resistors}

As explained before, the tightly regulated $\mathrm{MC}$ can be considered as a CPL as it has a negative incremental input resistance. A common method to overcome the issue is adding a damping resistor $\left(R_{d}\right)$ in parallel with the filter inductor for compensating the negative incremental resistance of the MC, and increasing the stability range $[9,10]$. The damping factor of the input filter is normally small $\left(\zeta=\frac{R_{f}}{2} \sqrt{\frac{C_{f}}{L_{f}}}\right)$ due to the negligible value of the input filter inductor resistance $R_{f}$. This results in undesirable oscillations of the converter input current due to the presence of harmonics close to the resonance frequency of the input filter. By adding the parallel

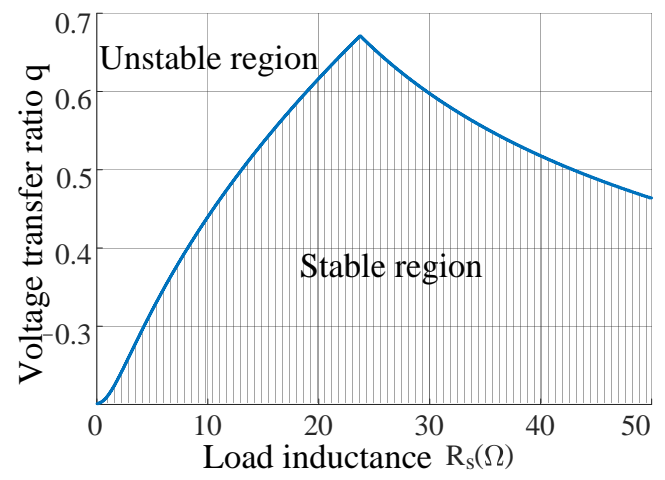

Fig. 3: The stable region of the system without applying any stabilization strategy, voltage gain $q$ against line resistance $R_{s}$

damping resistors, the cut-off frequency is almost the same as that of the LC filter $\left(\omega_{\text {coff }}=\frac{1}{\sqrt{L_{f} C_{f}}}\right)$ and the damping factor of the filter assuming $R_{f} \ll R_{d}$ is $\zeta_{d}=\frac{1}{2 R_{d}} \sqrt{\frac{L_{f}}{C_{f}}}$ [20]. To increase the damping property of the filter, $R_{d}$ should be reduced. However, smaller values of $R_{d}$ would result in higher power loss and larger THD of the converter input current which should be limited to the EMC requirements [24]. The high-frequency components of the input current flow through the damping resistor instead of the inductor during the transients and step changes of the input voltage which reduces the undesirable oscillations. To determine the stability limit of the converter, the linearized state space equations of the converter including the added damping resistor are defined as discussed in section 4 . The resultant state vector $X_{d}$ and the linearized state matrix $A_{d}$ can be presented as:

$$
\begin{aligned}
& \frac{d \Delta X_{d}}{d t}=A_{d} \Delta X_{d} \\
& X_{d}=\left[\begin{array}{llllllll}
i_{s i(d)} & i_{s i(q)} & v_{i(d)} & v_{i(q)} & i_{L f(d)} & i_{L f(q)} & i_{o(d)} & i_{o(q)}
\end{array}\right]^{T}
\end{aligned}
$$




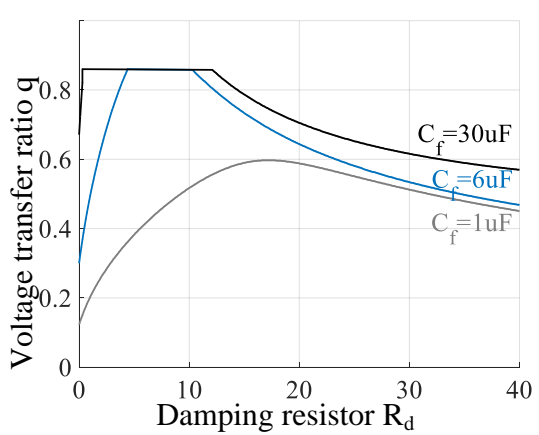

$a$

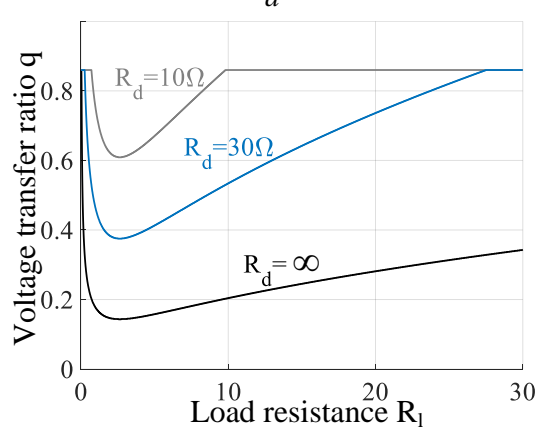

$d$

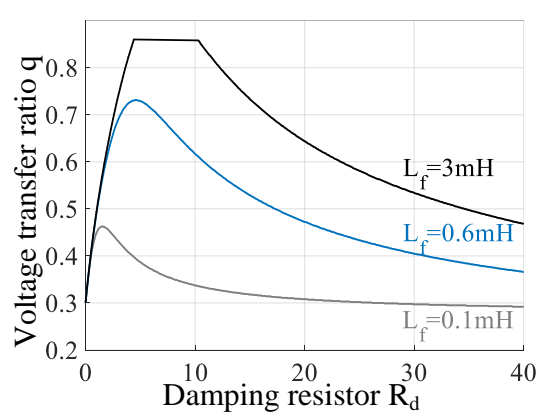

$b$

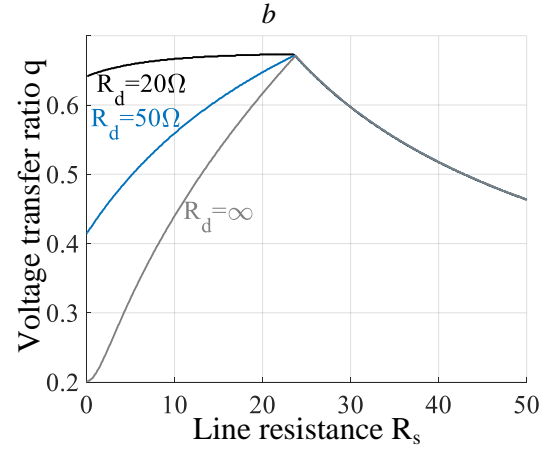

$e$

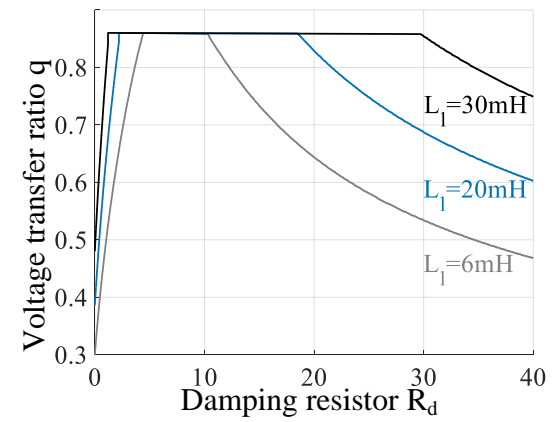

$c$

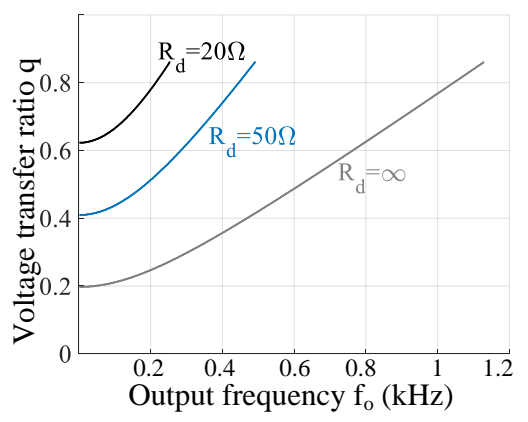

$f$

Fig. 4: The stable region of the MC system using the parallel damping resistor stabilization strategy, voltage gain 'q' against, (a) (b)(c) Damping resistor $R_{d}$ for different values of $C_{f}, L_{f}$ and $L_{l}$ respectively, $(d)$ Load resistance $R_{l},(e)$ Line resistance $R_{s},(f)$ Output frequency $f_{o}$, for different values of $R_{d}$

$$
\begin{aligned}
A_{d} & =\left[\begin{array}{cccccccc}
k & \omega_{i} & \frac{-1}{L_{s}} & 0 & \frac{R_{d}}{L_{s}} & 0 & 0 & 0 \\
-\omega_{i} & k & 0 & \frac{-1}{L_{s}} & 0 & \frac{R_{d}}{L_{s}} & 0 & 0 \\
\frac{1}{C_{f}} & 0 & k_{1} & \omega_{i} & 0 & 0 & -\frac{q}{C_{f}} & 0 \\
0 & \frac{1}{C_{f}} & -\omega_{i} & -k_{1} & 0 & 0 & 0 & 0 \\
\frac{R_{d}}{L_{f}} & 0 & 0 & 0 & -\frac{R_{d}}{L_{f}} & \omega_{i} & 0 & 0 \\
0 & \frac{R_{d}}{L_{f}} & 0 & 0 & -\omega_{i} & -\frac{R_{d}}{L_{f}} & 0 & 0 \\
0 & 0 & 0 & 0 & 0 & 0 & -\frac{R_{l}}{L_{l}} & \omega_{o} \\
0 & 0 & 0 & 0 & 0 & 0 & -\omega_{o} & -\frac{R_{l}}{L_{l}}
\end{array}\right] \\
k=-\frac{R_{s}+R_{d}}{L_{s}} &
\end{aligned}
$$

where $i_{L f(d)}$ and $i_{L f(q)}$ are the d-q components of the input filter inductor current $\vec{i}_{L f}$. Although, the state matrix $A_{d}$ is based on the voltage gain $q$, it can be seen as a function of the output power as has been presented in [10]. Figs. 4 and 5 show that all the system parameters can affect the position of the dominant eigenvalue and as a result, affect the system stability. While the curves determine the stability margin, the stability region is under the curves. Fig. 4a presents the limit of the voltage gain $q$ against the damping resistor $R_{d}$ for different values of $C_{f}$. With higher values of $C_{f}$, the maximum voltage gain is accessible with a wider range of $R_{d}$. The reason is that increasing the filter capacitance can reduce the voltage ripples, resulting in a higher voltage quality at the input of the MC which is used for calculating the duty cycles. On the other hand, as presented in Fig. 4b, increasing the filter inductance expands the stability region similarly, and increasing $L_{f}$ causes growing its reactance compared with the damping resistance. As a result, the stability improves as the high-frequency harmonics of the current mostly flows through the damping resistor instead of the inductor.

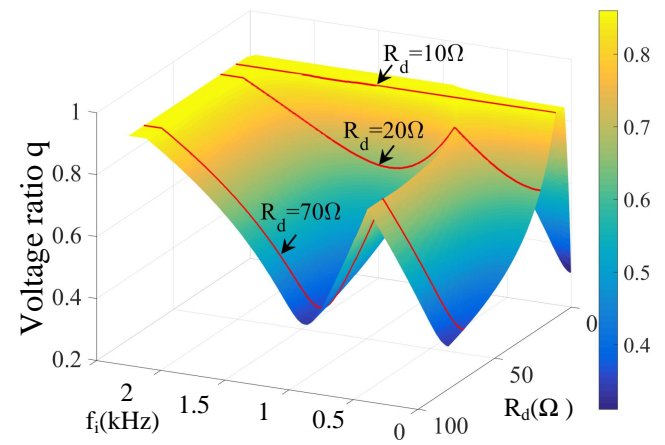

Fig. 5: The stable region of the MC system using the parallel damping resistor stabilization strategy, voltage gain 'q' against $R_{d}$ for different values of $f_{i}$

Also, as can be seen in Figs. $4 \mathrm{c}$ and $4 \mathrm{~d}$, increasing the load inductance $L_{l}$ or resistance $R_{l}$ expands the stability region and provides a wider range of $R_{d}$. Fig. $4 \mathrm{e}$ shows that the maximum voltage gain and stability is only possible for a limited value of series damping resistor $R_{s}$. On the other hand, high series damping resistance increases the power loss intensely. The effects of the output and input frequencies $f_{o}$ and $f_{i}$ have been illustrated in Figs. 4f and 5. In the case of $f_{o}$, for any specific value of $R_{d}$, increasing the frequency can increase the stability region. In the case of $f_{i}$, for small values of $R_{d}$, the inductor impedance is much more than the damping resistance and therefore, the effect of frequency is negligible. However, for large values of $R_{d}$, the effect of frequency on the stability is considerable, particularly at the input filter resonant frequency.

\section{Stabilization Technique Using the Input Voltage Digital Filter}

Active damping techniques act as a virtual resistor to damp the input filter by modifying the controller and overcome the negative 

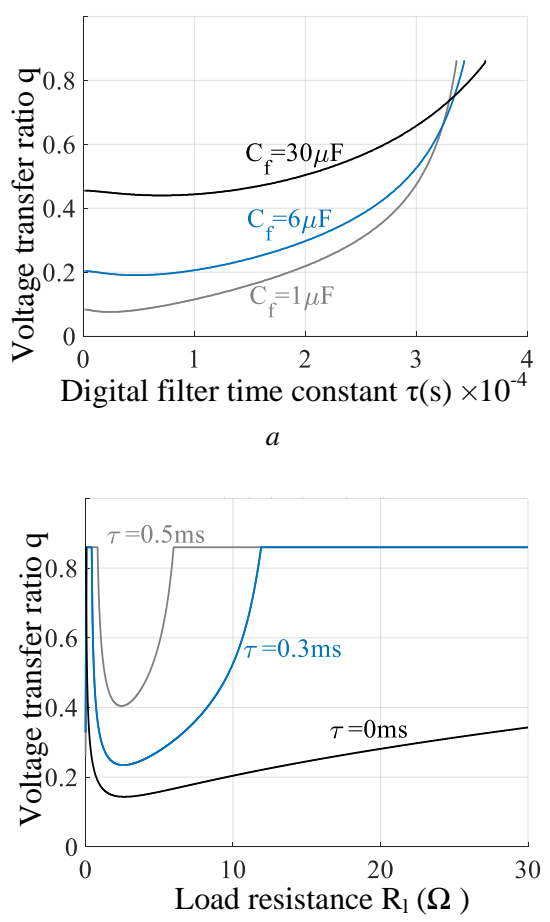

$d$

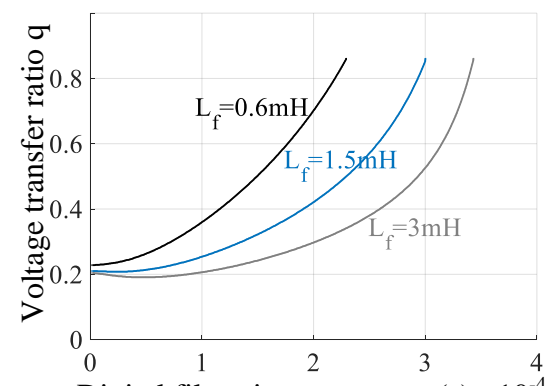

Digital filter time constant $\tau(\mathrm{s}) \times 10^{-4}$

$b$

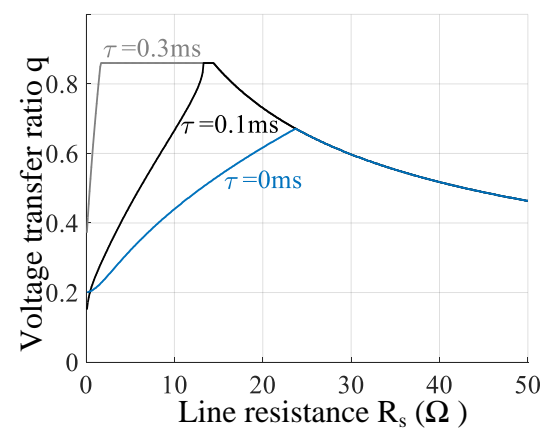

$e$
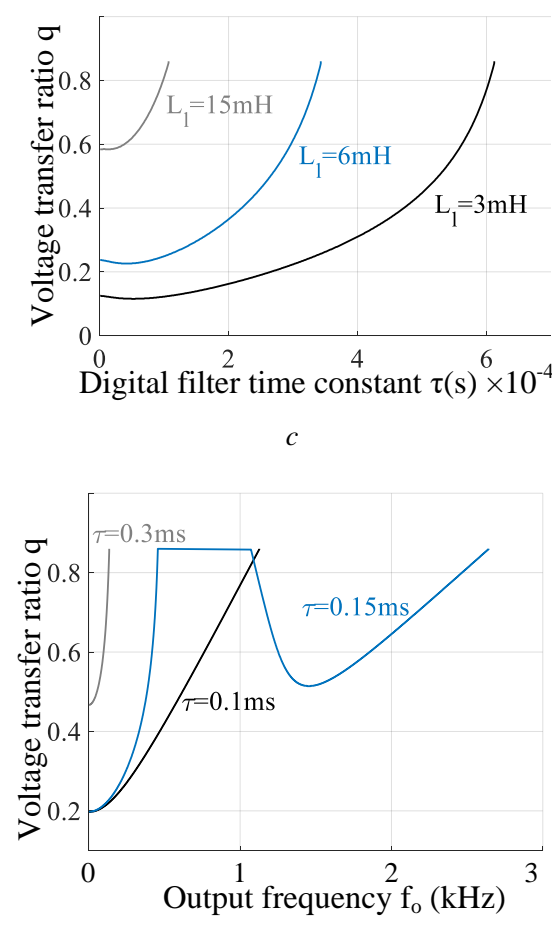

$f$

Fig. 6: The stable region of the MC system using the input voltage digital filter stabilization strategy, voltage gain 'q' against, (a) (b)(c) $\tau$ for different values of $C_{f}, L_{f}$ and $L_{l}$ respectively, $(d)$ Load resistance $R_{l},(e)$ Line resistance $R_{s}$, $(f)$ Output frequency $f_{o}$, for different values of $\tau$

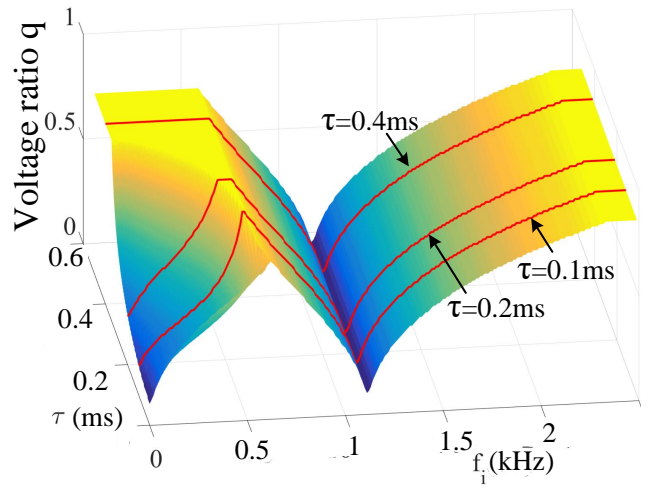

Fig. 7: The stable region of the MC system using the input voltage digital filter stabilization strategy, voltage gain 'q' against $\tau$ for different values of $f_{i}$

impedance instability problem caused by the tightly regulated converters $[7,8]$. The stabilization technique using a digital filter is an active method which is suggested in [13-15]. In this method, a lowpass digital filter with the time constant $\tau$ is implemented in the main processor to filter out the measured input voltage magnitude. The transfer function of the digital filter is a simple low-pass filter with a cut-off frequency $f_{c u t-o f f}=\frac{1}{2 \pi \tau}$. As an advantage, the method has no extra cost and power loss as it is implemented in a software form. It can filter out the steady state high-frequency harmonics of the input voltage around the resonant frequency of the input filter. However, there is a possibility for input voltage disturbances to be reflected on the output voltage proportional to the filter time constant [14]. The filter time constant can be increased to reduce the cut-off frequency and attenuate the low-frequency harmonics. This can cause more high-frequency harmonics of the input voltage transfer to the output side. Furthermore, the transient oscillations at the time of a power step are the other negative aspects of this strategy. The linearized state matrix of the converter assuming a digital filter with time constant $\tau$ is obtained as [14]:

$$
\begin{aligned}
& \frac{d \Delta X_{f}}{d t}=A_{f} \Delta X_{f} \\
& X_{f}=\left[\begin{array}{ccccccccc}
i_{s i(d)} & i_{s i(q)} & v_{i(d)} & v_{i(q)} & i_{o(d)} & i_{o(q)} & v_{i f(d)} & \left.v_{i f(q)}\right]^{T} & (10) \\
A_{f}= & {\left[\begin{array}{cccccccc}
-\frac{R_{s}}{L_{T}} & \omega_{i} & \frac{-1}{L_{T}} & 0 & 0 & 0 & 0 & 0 \\
-\omega_{i} & -\frac{R_{s}}{L_{T}} & 0 & \frac{-1}{L_{T}} & 0 & 0 & 0 & 0 \\
\frac{1}{C_{f}} & 0 & 0 & \omega_{i} & -\frac{q}{C_{f}} & 0 & k_{1} & 0 \\
0 & \frac{1}{C_{f}} & -\omega_{i} & 0 & 0 & 0 & 0 & -k_{1} \\
0 & 0 & \frac{q}{L_{l}} & 0 & -\frac{R_{l}}{L_{l}} & \omega_{o} & -\frac{q}{L_{l}} & 0 \\
0 & 0 & 0 & 0 & -\omega_{o} & -\frac{R_{l}}{L_{l}} & 0 & 0 \\
0 & 0 & \frac{1}{\tau} & 0 & 0 & 0 & -\frac{1}{\tau} & 0 \\
0 & 0 & 0 & \frac{1}{\tau} & 0 & 0 & 0 & -\frac{1}{\tau}
\end{array}\right]}
\end{array}\right.
\end{aligned}
$$

where $v_{i f(d)}$ and $v_{i f(q)}$ are the d-q components of the filtered input voltage vector $\vec{v}_{i f}$. The stability margins of the MC are shown in Figs. 6 and 7 for different values of the system parameters and $\tau$. The areas under the curves and the 3D-plane are the stability regions. As can be seen, depending on the system parameters there is a spacial $\tau$ for achieving the maximum voltage gain. In general, similar to the damping resistor method, with larger values of $C_{f}$, a wider stability region and higher voltage gains are achievable for a certain value of $\tau$ as can be seen in Fig. 6 a. In contrast to $C_{f}$, a smaller $L_{f}$ can reduce the required $\tau$ for the maximum voltage gain, as illustrated in Fig. $6 \mathrm{~b}$. Figs. $6 \mathrm{c}$ and $6 \mathrm{~d}$ show the effects of the RL load which increment of the $R_{l}$ and $L_{l}$ improves the stability. In the case of $R_{l}$, reducing the resistance leads to a reduction of the voltage gain limit, but for the small values of $R_{l}$ the maximum voltage gain is accessible by using a proper $\tau$. Like the damping resistor method, larger values of $R_{s}$ expands the stability region, but depending on the system 


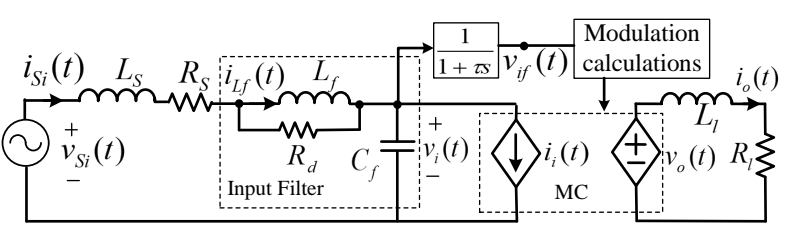

Fig. 8: Block diagram of a single-phase with the digital low-pass filter and damping resistor

parameters and $\tau$, the voltage gain limit starts to reduce at a spacial point as shown in Fig. 6e. The effect of increasing $f_{o}$ on improving the stability region is shown in Fig. 6f that depending on the system parameters for some values of $\tau$, the voltage gain limit starts to decrease in a special $f_{o}$, but again by increasing the frequency, it increases to reach the maximum voltage gain. The 3D graph in Fig. 7 shows the stability border with varying $f_{i}$ for different values of $\tau$. Similar to the damping resistor method, by increasing the frequency at first the voltage gain limit increases, then it starts to reduce until in the resonant frequency of the input filter it gets to the first minimum voltage gain, and after that, it begins to increase again.

\section{Developing Mathematical Model for Stability Analysis Using the Combination Method}

The third stabilization technique proposed in this paper is based on a combination of the previously discussed techniques as presented in Fig. 8. The resultant method takes the advantages of both damping resistor and digital filter methods while minimizes their drawbacks. In this method, the converter stability for the entire operating range is achieved using higher damping resistances and consequently less power loss. In addition, the undesirable oscillations during the step changes of the voltage or current can be avoided. Finally, the input and output currents are sinusoidal with an acceptable THD for the entire operating range.

The stability analysis of MC using the combination method is presented in this section. Firstly, the state equations of the system in the synchronous reference frame rotating at the input source angular frequency $\left(\omega_{i}\right)$ are obtained as following (referring to Fig. 8):

$$
\begin{gathered}
\frac{d \vec{i}_{s i}(t)}{d t}=-\left(\frac{R_{s}+R_{d}}{L_{s}}+j \omega_{i}\right) \vec{i}_{s i}(t)+\frac{1}{L_{s}} \vec{v}_{s i}(t)-\frac{1}{L_{s}} \vec{v}_{i}(t)+\frac{R_{d}}{L_{s}} \vec{i}_{L f}(t) \\
\frac{d \vec{v}_{i}(t)}{d t}=\frac{1}{C_{f}} \vec{i}_{s i}(t)-j \omega_{i} \vec{v}_{i}(t)-\frac{1}{C_{f}} \vec{i}_{i}(t) \\
\frac{d \vec{i}_{L f}(t)}{d t}=\frac{R_{d}}{L_{f}} \vec{i}_{s i}(t)-\left(\frac{R_{d}}{L_{f}}+j \omega_{i}\right) \vec{i}_{L f}(t) \\
\frac{d \vec{v}_{i f}(t)}{d t}=\frac{1}{\tau} \vec{v}_{i}(t)-\frac{1}{\tau} \vec{v}_{i f}(t)
\end{gathered}
$$

where $\vec{v}_{s i}$ and $\vec{i}_{s i}$ are the input source voltage and current space vectors, $\vec{v}_{i}$ and $\vec{i}_{i}$ the input voltage and current to the MC, respectively, and $\vec{i}_{l f}$ is the current in the input filter inductor. The state equation in the output side can be defined similarly in the synchronous reference frame rotating at $\omega_{o}$ as:

$$
\frac{d \vec{i}_{o}(t)}{d t}=-\left(\frac{R_{l}}{L_{l}}+j \omega_{o}\right) \vec{i}_{o}(t)+\frac{1}{L_{l}} \vec{v}_{o}(t)
$$

where $\vec{v}_{o}$ and $\vec{i}_{o}$ are the output voltage and current, respectively. The relation between the input and output of the MC can be defined based on the output voltage and input current as suggested in [25] from:

$$
\begin{gathered}
\vec{v}_{o}(t)=\frac{3}{2}\left[\vec{v}_{i f}(t) \vec{m}_{i}^{*}(t)+\vec{v}_{i f}^{*}(t) \vec{m}_{d}(t)\right] \\
\vec{i}_{i}(t)=\frac{3}{2}\left[\vec{i}_{o}(t) \vec{m}_{i}(t)+\vec{i}_{o}^{*}(t) \vec{m}_{d}(t)\right]
\end{gathered}
$$

where $\vec{m}_{d}(t)$ and $\vec{m}_{i}(t)$ are the complex variables known as the duty cycles space vectors and are determined based on the output voltage reference vector $\vec{v}_{\text {or }}$, input voltage from the digital filter, $\vec{v}_{i f}$ and the arbitrary space vector $\vec{\psi}(t)$ from:

$$
\vec{m}_{i}(t)=\frac{\vec{v}_{o r}^{*}(t) \vec{\psi}(t)}{3\left[\vec{v}_{i f}(t) \cdot \vec{\psi}(t)\right]}, \vec{m}_{d}(t)=\frac{\vec{v}_{o r}(t) \vec{\psi}(t)}{3\left[\vec{v}_{i f}(t) \cdot \vec{\psi}(t)\right]}
$$

where the symbol '*, represents the complex conjugate of the vector. To generate an input current vector with the desired angle between input current and voltage space vectors, it should be modulated along with the vector $\vec{\psi}(t)$ as [10][26]:

$$
\vec{i}_{i}(t) \cdot j \vec{\psi}(t)=0
$$

To realize a unity input power factor, the input current should be modulated along with the input voltage space vector. Therefore, the arbitrary vector $\vec{\psi}(t)$ is defined as:

$$
\vec{\psi}(t)=\vec{v}_{i f}(t) e^{-j \varphi_{i}}
$$

where $\varphi_{i}$ is the displacement angle between the input voltage and current space vectors and should be equal to zero for unity input power factor which results in $\vec{\psi}(t)=\vec{v}_{i f}(t)$. The duty cycles space vectors $\vec{m}_{i}(t)$ and $\vec{m}_{d}(t)$ presented in (19) then are simplified accordingly as $[13,14]$ :

$$
\vec{m}_{i}(t)=\frac{\vec{v}_{o r}^{*}(t)}{3 \vec{v}_{i f}^{*}(t)}, \vec{m}_{d}(t)=\frac{\vec{v}_{o r}(t)}{3 \vec{v}_{i f}^{*}(t)}
$$

The resultant equations are used in the following section to obtain the small-signal model of the converter based on the method presented in $[10,14]$.

\subsection{Small Signal Model and Steady-State Operating Conditions}

The fundamental model of the power electronic converters is nonlinear in nature, however, it can be linearized around the steady state operating point for stability analysis. The reason is that stability of the linearized system at the steady state operating point guarantees the stability of the nonlinear system on the same operating point according to the Poincaré theorem [23]. The input and output voltages of the converter for the steady state operating condition can be written as:

$$
\left\{\begin{array}{l}
\vec{v}_{i}(t)=\vec{V}_{i} e^{j \omega_{i} t}=V_{i m} e^{j \alpha_{i}} e^{j \omega_{i} t} \\
\vec{v}_{o r}(t)=\vec{V}_{o r} e^{j \omega_{o} t}=V_{o r} e^{j \alpha_{o}} e^{j \omega_{o} t}
\end{array}\right.
$$

where $\alpha_{i}$ and $\alpha_{o}$ are the angles of the input and output reference voltages respectively. Assuming that $\vec{v}_{i}(t)$ and $\vec{v}_{o r}(t)$ are along the $\mathrm{d}$-axis, then $\alpha_{i}=\alpha_{o}=0$. So (23) can be simplified as:

$$
\left\{\begin{array}{l}
\vec{v}_{i}(t)=V_{i m} e^{j \omega_{i} t} \\
\vec{v}_{o r}(t)=V_{o r} e^{j \omega_{o} t}
\end{array}\right.
$$

In the steady state operating point, all variables with their specific synchronous reference frame and angular frequency can be considered as constant values. Therefore, the system variables at the steady 
state operating point are presented as [14]:

$$
\begin{aligned}
& \vec{V}_{i}=V_{i m}, \vec{V}_{o r}=V_{o r} \Rightarrow \vec{V}_{i f}=V_{i m}, q=\frac{V_{o r}}{V_{i m}}, \\
& \vec{M}_{d}=\frac{q}{3}, \vec{M}_{i}=\frac{q}{3}, \vec{I}_{o}=\frac{q V_{i m}}{\vec{Z}_{l}}
\end{aligned}
$$

The small-signal model of the system can be found by applying the small perturbations to the system variables at their steady-state values. Assuming the input voltage and output reference voltage are sinusoidal with almost constant amplitudes, one has $\Delta \vec{v}_{s i}(t)=0$ and $\Delta \vec{v}_{o r}(t)=0$. Therefore, the variable components of $\vec{m}_{i}(t)$ and $\vec{m}_{d}(t)$, input current $\vec{i}_{i}(t)$ and output voltage $\vec{v}_{o}(t)$ by using (22), (17) and (18) are defined as:

$$
\begin{aligned}
& \begin{aligned}
\Delta \vec{m}_{d}(t) & =\frac{1}{3} \frac{\Delta \vec{v}_{o r}(t) \vec{V}_{i f}^{*}-\vec{V}_{o r} \Delta \vec{v}_{i f}^{*}(t)}{\left(\vec{V}_{i f}^{*}\right)^{2}}=-\frac{q}{3 V_{i m}} \Delta \vec{v}_{i f}^{*}(t) \\
\Delta \vec{m}_{i}(t) & =\frac{1}{3} \frac{\Delta \vec{v}_{o r}^{*}(t) \vec{V}_{i f}^{*}-\vec{V}_{o r}^{*} \Delta \vec{v}_{i f}^{*}(t)}{\left(\vec{V}_{i f}^{*}\right)^{2}}=-\frac{q}{3 V_{i m}} \Delta \vec{v}_{i f}^{*}(t) \\
\Delta \vec{v}_{o}(t) & =\frac{3}{2}\left[\Delta \vec{v}_{i}(t) \vec{M}_{i}^{*}+\Delta \vec{v}_{i}^{*}(t) \vec{M}_{d}+V_{i m} \Delta \vec{m}_{i}^{*}(t)+V_{i m} \Delta \vec{m}_{d}(t)\right] \\
& =\frac{q}{2} \Delta \vec{v}_{i}(t)+\frac{q}{2} \Delta \vec{v}_{i}^{*}(t)-\frac{q}{2} \Delta \vec{v}_{i f}(t)-\frac{q}{2} \Delta \vec{v}_{i f}^{*}(t)
\end{aligned} \\
& \Delta \vec{i}_{i}(t)=\frac{3}{2}\left[\Delta \vec{i}_{o}(t) \vec{M}_{i}+\Delta \vec{i}_{o}^{*}(t) \vec{M}_{d}+\vec{I}_{o} \Delta \vec{m}_{i}(t)+\vec{I}_{o}^{*} \Delta \vec{m}_{d}(t)\right] \\
& =\frac{q}{2} \Delta \vec{i}_{o}(t)+\frac{q}{2} \Delta \vec{i}_{o}^{*}(t)-\frac{q^{2} R_{l}}{\left|\vec{Z}_{l}\right|^{2}} \Delta \vec{v}_{i f}^{*}(t)
\end{aligned}
$$

To facilitate the controller design, (28) and (29) are decomposed into their equivalent d-q components as:

$$
\begin{gathered}
\left\{\begin{array}{l}
\Delta v_{o(d)}(t)=q \Delta v_{i(d)}(t)-q \Delta v_{i f(d)}(t) \\
\Delta v_{o(q)}(t)=0
\end{array}\right. \\
\left\{\begin{array}{l}
\Delta i_{i(d)}(t)=q \Delta i_{o(d)}(t)-\frac{q^{2} R_{l}}{\left|\vec{Z}_{l}\right|^{2}} \Delta v_{i f(d)}(t) \\
\Delta i_{i(q)}(t)=\frac{q^{2} R_{l}}{\left|\vec{Z}_{l}\right|^{2}} \Delta v_{i f(q)}(t)
\end{array}\right.
\end{gathered}
$$

To find the final state equations of the system, (12)-(16) are linearized around the steady-state operating point, and decomposed into their d-q components. Substituting (30) and (31), one obtains the resultant equations as the following:

$$
\left\{\begin{array}{l}
\frac{d \Delta v_{i(d)}(t)}{d t}=\frac{1}{C_{f}} \Delta i_{s i(d)}(t)+\omega_{i} \Delta v_{i(q)}(t)-\frac{q}{C_{f}} \Delta i_{o(d)}(t)+\frac{q^{2} R_{l}}{C_{f}\left|\vec{Z}_{l}\right|^{2}} \Delta v_{i f(d)}(t) \\
\frac{d \Delta v_{i(q)}(t)}{d t}=\frac{1}{C_{f}} \Delta i_{s i(q)}(t)-\omega_{i} \Delta v_{i(d)}(t)-\frac{q^{2} R_{l}}{C_{f}\left|\vec{Z}_{l}\right|^{2}} \Delta v_{i f(q)}(t)
\end{array}\right.
$$

$$
\left\{\begin{array}{l}
\frac{d \Delta i_{o(d)}(t)}{d t}=\frac{q}{L_{l}} \Delta v_{i(d)}(t)-\frac{R_{l}}{L_{l}} \Delta i_{o(d)}(t)+\omega_{o} \Delta i_{o(q)}(t)-\frac{q}{L_{l}} \Delta v_{i f(d)}(t) \\
\frac{d \Delta i_{o(q)}(t)}{d t}=-\omega_{o} \Delta i_{o(d)}(t)-\frac{R_{l}}{L_{l}} \Delta i_{o(q)}(t)
\end{array}\right.
$$

$$
\left\{\begin{array}{l}
\frac{d \Delta v_{i f(d)}(t)}{d t}=\frac{1}{\tau} \Delta v_{i(d)}(t)-\frac{1}{\tau} \Delta v_{i f(d)}(t) \\
\frac{d \Delta v_{i f(q)}(t)}{d t}=\frac{1}{\tau} \Delta v_{i(q)}(t)-\frac{1}{\tau} \Delta v_{i f(q)}(t)
\end{array}\right.
$$

The equations can be rewritten in the matrix form to facilitate the stability analysis as:

$$
\frac{d \Delta X_{c}}{d t}=A_{c} \Delta X_{c}
$$

where state vector $\Delta X_{c}$ and the linearized state matrix $A_{c}$ are defined as:

$$
\begin{aligned}
X_{c} & =\left[\begin{array}{cccccccccc}
i_{s i(d)} & i_{s i(q)} & v_{i(d)} & v_{i(q)} & i_{L f(d)} & i_{L f(q)} & v_{i f(d)} & v_{i f(q)} & i_{o(d)} & i_{o(q)}
\end{array}\right]^{T} \\
A_{c} & =\left[\begin{array}{cccccccccc}
k & \omega_{i} & -\frac{1}{L_{s}} & 0 & \frac{R_{d}}{L_{s}} & 0 & 0 & 0 & 0 & 0 \\
-\omega_{i} & k & 0 & -\frac{1}{L_{s}} & 0 & \frac{R_{d}}{L_{s}} & 0 & 0 & 0 & 0 \\
\frac{1}{C_{f}} & 0 & 0 & \omega_{i} & 0 & 0 & k_{1} & 0 & -\frac{q}{C_{f}} & 0 \\
0 & \frac{1}{C_{f}} & -\omega_{i} & 0 & 0 & 0 & 0 & -k_{1} & 0 & 0 \\
\frac{R_{d}}{L_{f}} & 0 & 0 & 0 & -\frac{R_{d}}{L_{f}} & \omega_{i} & 0 & 0 & 0 & 0 \\
0 & \frac{R_{d}}{L_{f}} & 0 & 0 & -\omega_{i} & -\frac{R_{d}}{L_{f}} & 0 & 0 & 0 & 0 \\
0 & 0 & \frac{1}{\tau} & 0 & 0 & 0 & -\frac{1}{\tau} & 0 & 0 & 0 \\
0 & 0 & 0 & \frac{1}{\tau} & 0 & 0 & 0 & -\frac{1}{\tau} & 0 & 0 \\
0 & 0 & \frac{q}{L_{l}} & 0 & 0 & 0 & -\frac{q}{L_{l}} & 0 & -\frac{R_{l}}{L_{l}} & \omega_{o} \\
0 & 0 & 0 & 0 & 0 & 0 & 0 & 0 & -\omega_{o} & -\frac{R_{l}}{L_{l}}
\end{array}\right]
\end{aligned}
$$

Similar to the previously discussed methods, matrix $A_{c}$ and its eigenvalues can be effectively used to determine the system stability conditions. As can be seen in (36), the eigenvalues of matrix $A_{c}$ are a function of the damping resistor $R_{d}$, the digital filter time constant $\tau$ and the other parameters of the system.

Fig. 9a illustrates the stability region of the converter based on the voltage gain $q$ and damping resistor $R_{d}$ for different values of $\tau$. As can be seen, the stability region with the maximum gain increases for higher time constants and damping resistors. However, higher values of $\tau$ reduce the digital filter cutoff frequency which is not desirable. Therefore, the selection of proper damping resistor and filter time constant remains a trade-off between damping power loss and EMC requirements. Fig. $9 \mathrm{~b}$ presents the stability region based on the time constant for different damping resistors. As can be seen, the higher voltage gain, wider stability region, and larger damping resistor are achievable at higher time constants.

\section{Assessment of the MC Stability for Bidirectional Power-Flow Control}

To compare the characteristics of the discussed stabilization techniques, a DMC with four-quadrant bidirectional power flow control is numerically simulated. The converter is used as an interface between the utility grid and a microgrid with common ac bus as presented in Fig. 10. As can be seen, the three-phase voltage source $v_{s}(t)$ at the input side represents the utility grid and $v_{o}(t)$ the ac microgrid. A voltage oriented control (VOC) technique [27, 28] using anti-windup proportional integral (PI) compensators is implemented to generate the required drive signals. The space vector modulation (SVM) technique with a double-sided switching pattern is used to control the switching durations with minimum switching losses. The d-q components of the output current are used to control the active and reactive power and power flow direction. The power flow is controlled at one side as simultaneous double sided power flow control is not possible due to the lack of energy storage device in the MC topology. The stability of the converter is important in the steady conditions and transients when a step change in the amount or direction of the output power is applied. To compare the stabilization techniques, several indicators such as maximum voltage gain, THD of the input and output currents, power up performance have been taken into account. 


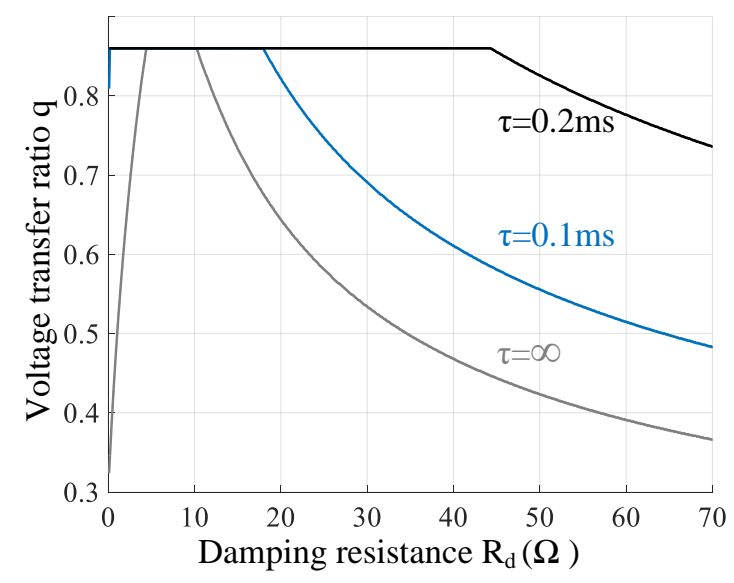

$a$

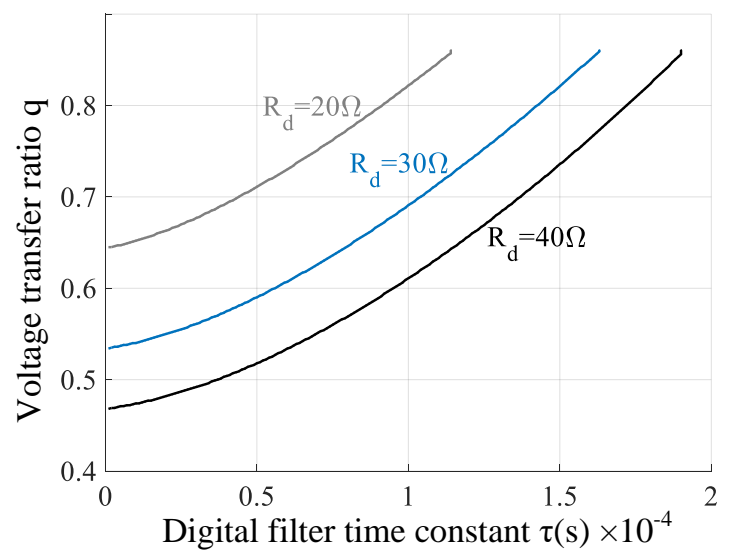

$b$

Fig. 9: The stable region of the MC system using a combination of the damping resistor and the input voltage digital filter stabilization methods,

(a) Voltage gain 'q' against $R_{d}$ for different values of $\tau$, (b) Voltage gain 'q' against $\tau$ for different values of $R_{d}$

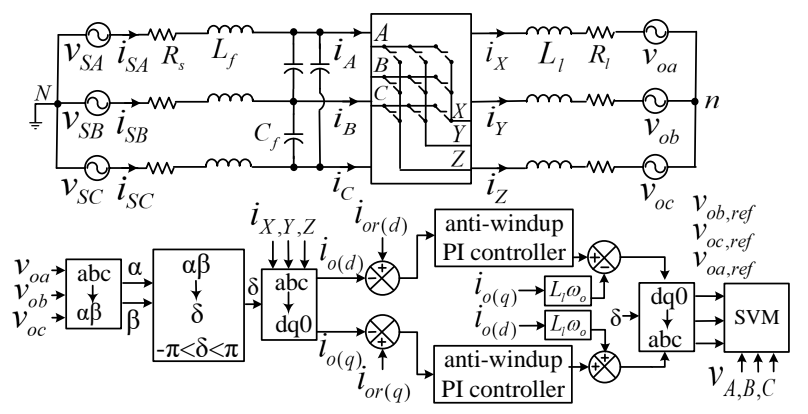

Fig. 10: System diagram for bidirectional active and reactive output power flow control

\section{Simulation Results}

The proposed DMC system is simulated using PSIM for the application presented in Fig. 10. The purpose of the simulation and the experimental tests is not to show the stability borders, but to illustrate the performance of the stabilization methods in different aspects like transients and the input current THD. Operation of the converter for the maximum voltage transfer ratio $(q=0.866)$ in the experimental tests is difficult to be achieved, as it needs fine-tuning of the voltage and current sensors, and precise regulation of the coefficients in the DSP. As illustrated in the above mentioned numerical analysis, the

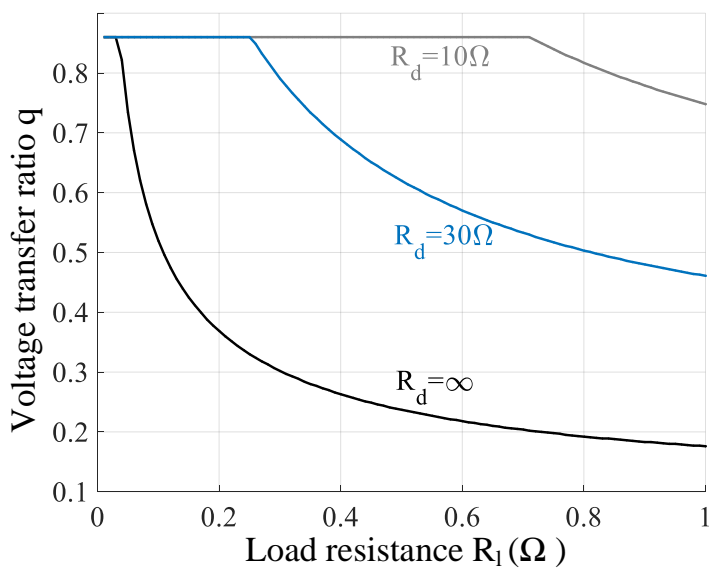

Fig. 11: The stable region of the MC system using the parallel damping resistor stabilization strategy, voltage gain 'q' against $R_{l}$ for different values of $R_{d}$

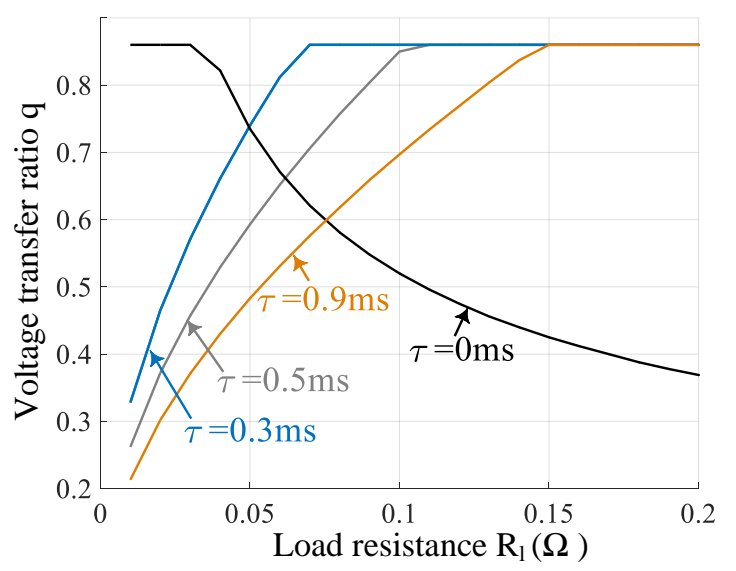

Fig. 12: The stable region of the MC system using the input voltage digital filter stabilization strategy, voltage gain 'q' against $R_{l}$ for different values of $\tau$

Table 1 System parameters for power-flow control simulations

\begin{tabular}{ll}
\hline Input source voltage & $400 \mathrm{~V}, f_{i}=50 \mathrm{~Hz}$ \\
Output source voltage & $230 \mathrm{~V}, f_{o}=70 \mathrm{~Hz}$ \\
Input line impedance & $L_{s}=0.4 \mathrm{mH}, R_{s}=0.5 \Omega$ \\
Input filter & $L_{f}=0.6 \mathrm{mH}, C_{f}=12.6 \mu \mathrm{F}$ \\
Switching frequency & $f_{s}=10 \mathrm{kHz}$ \\
output filter & $L_{l}=6 \mathrm{mH}$ \\
output resistance & $R_{l}=0.1 \Omega$ \\
Simulation time step & $1 \mu s$ \\
\hline
\end{tabular}

system stability can be affected by the output resistance. Figs. $2 \mathrm{f}$, $4 \mathrm{~d}$ and $6 \mathrm{~d}$ show that the stability of the system with the maximum gain is achievable in larger load resistances, but the graphs show a different trend for the small resistances. In case of the bidirectional power flow which the converter output is connected to another threephase source, $R_{l}$ can represent the microgrid line resistance which is very small. In the simulation it is assumed $0.1 \Omega$. To compare the stabilization methods in this range of the resistance, Figs. 11 and 12 have focused on the resistances under $1 \Omega$. As can be seen, in the damping resistor strategy, by reducing the damping resistance the stability is achievable for higher voltage gains, while using the input digital filter the stability can not be guaranteed by increasing the $\tau$. In the bidirectional simulation tests with the same system parameters in section 3 when $R_{l}=0.1 \Omega$ the system stays stable using the damping resistor $R_{d}=3 \Omega$ in any voltage gain. But, using the input digital filter, the stabilization is possible only for low voltage 

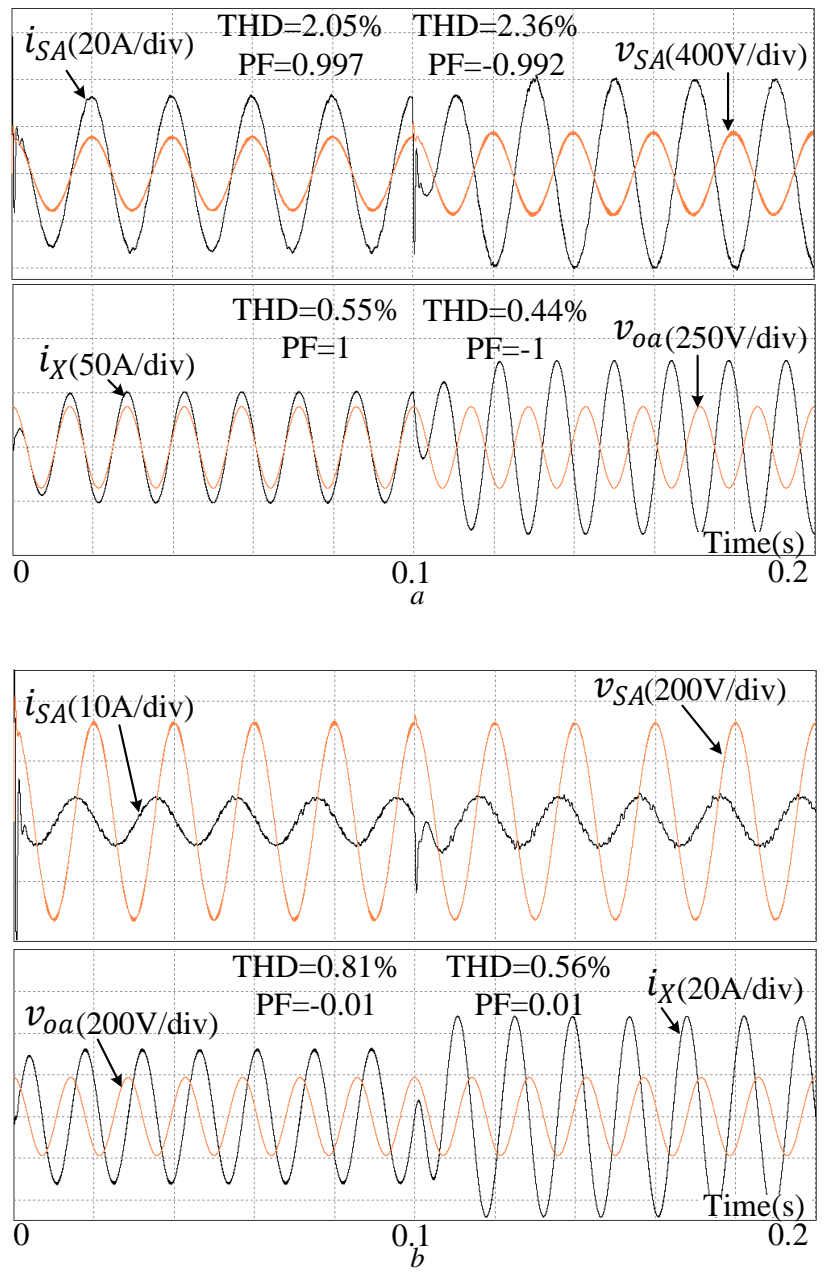

Fig. 13: The input source current and output current for bidirectional power-flow control, using damping resistor $R_{d}=3 \Omega$,

(a) Bidirectional active power-flow control, (b) Bidirectional reactive power-flow control

gains. On the other hand, as mentioned before in Fig. 6b, reducing the $L_{f}$ helps the system stability in the digital filter stabilization method. Indeed, to keep the cut-off frequency in the suitable range, $C_{f}$ has to be increased. By this way, the problem related to the small $R_{l}$ can also be solved. To show the system behaviour for all three stabilization techniques, $L_{f}$ is reduced to $0.6 \mathrm{mH}$ and to keep the cutoff frequency in the acceptable range $C_{f}$ has to be increased which $C_{f}=12.6 \mu \mathrm{F}$ has been selected for this purpose $\left(f_{c}=1830 \mathrm{~Hz}\right)$.
The simulated system parameters are listed in Table 1 . In the first time interval $0-0.1 s$ the power flows from the input to the output side of the converter and for the second time interval $0.1-0.2 s$ the power flows in the opposite direction from output to the input side. For testing the bidirectional active power flow from the input to the output side, the output current $d$ component is adjusted to $i_{o r(d)}=$ $51 A$ (peak) which presents the voltage gain about $q=0.75$. To control the active power direction from the output to the input, during the time $0.1-0.2 s, i_{\text {or }(d)}$ steps to $-80 A$ (peak) at $t=0.1 \mathrm{~s}$ that the voltage gain is about $q=0.82$. In purpose of the reactive power control, $i_{\text {or }(q)}$ at first is $32 \mathrm{~A}$ (peak) during the time interval $0-0.1 \mathrm{~s}$ with voltage gain $q=0.83$, and for the opposite reactive power flow direction, it steps to $i_{\text {or }(q)}=-48 A$ (peak) at $t=0.1 \mathrm{~s}$ which the voltage gain is almost $q=0.2$.

The simulation results for the damping resistor method with the small damping resistance $R_{d}=3 \Omega$ are shown in Fig. 13. It can be seen that the system is stable with low THDs of the input and output currents, and the oscillation at the transient times have been damped perfectly, although, this small resistance causes the efficiency to be reduced. Fig. 15 presents the same results for the input digital filter strategy with a time constant $\tau=0.5 \mathrm{~ms}$. As shown, the input current in the transient period oscillates a lot. Also, the THD of the input current is a little more than the previous stabilization method.

Fig. 16 shows the input and output currents and voltages for the combination method when $R_{d}=10 \Omega$, and $\tau=0.2 \mathrm{~ms}$. By increasing the damping resistance to $10 \Omega$ the average power loss reduces to about one third. The THDs of the currents are still less than that obtained by using only the digital filter, and the transient oscillations at the current step times are very close to the damping resistor method results. Also, despite increasing the damping resistance and reducing the digital filter time constant, the converter remains stable for the whole range of the voltage transfer ratio. However, the proper selection of stabilizing parameters ( $\tau$ and $R_{d}$ ) is a tradeoff between the acceptable ranges of the power-loss and transient oscillations.

\section{Experimental Results}

As there is no power source except for the utility grid, the power circuit suggested in Fig. 14 provides two isolated power sources with adjustable amplitudes but the same frequency using the isolating and variable transformers. The input source phase voltages are adjusted to $72 \mathrm{~V}$-rms and the output to $43 \mathrm{~V}$-rms with frequency of $50 \mathrm{~Hz}$. The applied voltages and currents in this section are based on the current limits of the components like the resistors and reactors used in the input filter and load. The switching frequency is $f_{s}=10 \mathrm{kHz}$ and the input filter parameters are $C_{f}=6.6 \mu \mathrm{F}$ and $L_{f}=3 \mathrm{mH}$. The inductance $L_{l}=6 \mathrm{mH}$ is used as the output filter.

In the practical control circuit, the anti-windup PI controllers are used due to the clock frequency effect of the field programable gate array (FPGA), to help stabilization of the system in high voltage

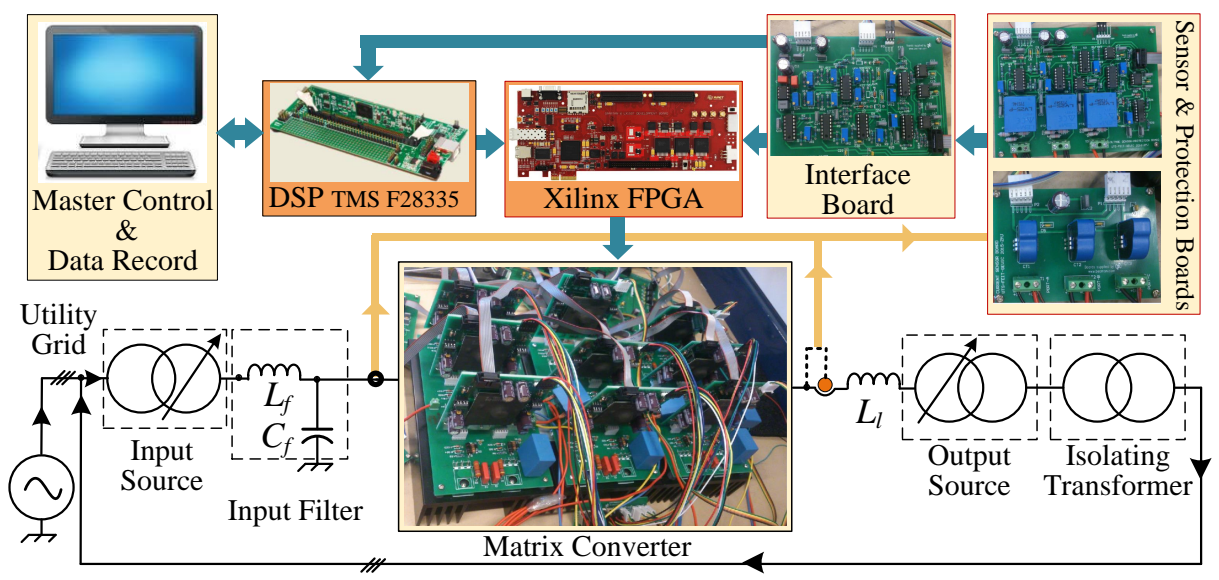

Fig. 14: Schematic of the designed system for the experimental test of the MC bidirectional power flow using utility grid 

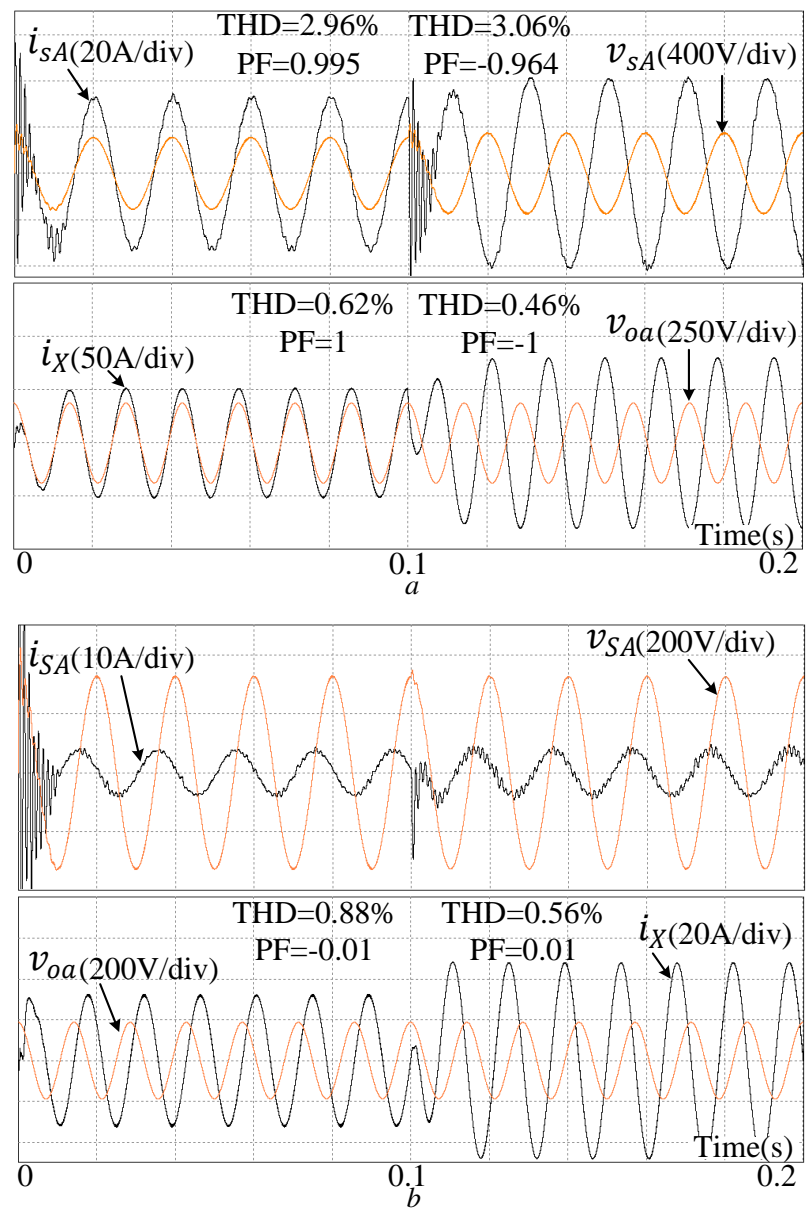

Fig. 15: The input source current and output current for bidirectional power-flow control, using the digital filter only with time constant $\tau=0.5 \mathrm{~ms}$,

(a) Bidirectional active power-flow control, (b) Bidirectional reactive power-flow control

gains. Without applying any stabilization method, the instability of the system can be observed for a voltage transfer ratio of about $q=0.75$ in Fig. 17a. In this test, the $d$ component of the output reference current $\left(i_{o r(d)}\right)$ changes from $4 A$ to $8 A$ (peak). Fig. 17b shows that the system is stable for $i_{o r(d)}=8 A$ when the large damping resistor $R_{d}=47 \Omega$ is placed in parallel with the filter inductor although the input current has high THD. Fig. 18 presents the experimental results in the case of using the combination method with $R_{d}=47 \Omega$ and digital filter time constant $\tau=0.2 \mathrm{~ms}$. The input and output currents for bidirectional active power control are shown in Fig. 18a when the d-component of the output reference current $i_{\text {or }(d)}$ is adjusted to change from $8 A$ to $-8 A$ (peak). The input and output power factors are almost one at the first stage which means the active power flows from input to the output, and it gets to almost -1 when the current flows from output to the input side of the converter. The same results are presented for bidirectional reactive power control by changing $i_{\text {or }(q)}$ from $8 A$ to $-8 A$ (peak) in Fig. 18b. As can be seen, the phase of the current is lagging the phase of the voltage by about $90^{\circ}$, and then it leads the output voltage. The same results are illustrated in Fig. 19 for the digital filter method with $\tau=0.5 \mathrm{~ms}$ and in Fig. 20 for the damping resistor method with a small damping resistance $R_{d}=3 \Omega$. The slight difference in the amplitude of the output voltage before and after the transient time is because of the difference in the employed structure of the voltage source at the input and output sides, as illustrated in Fig. 14. A comparison of the experimental results shows that the proposed combination method presents less input source current distortion when the active power is controlled to flow from the output to the input side. Considering the effect of the damping resistor on the efficiency of the converter, in the
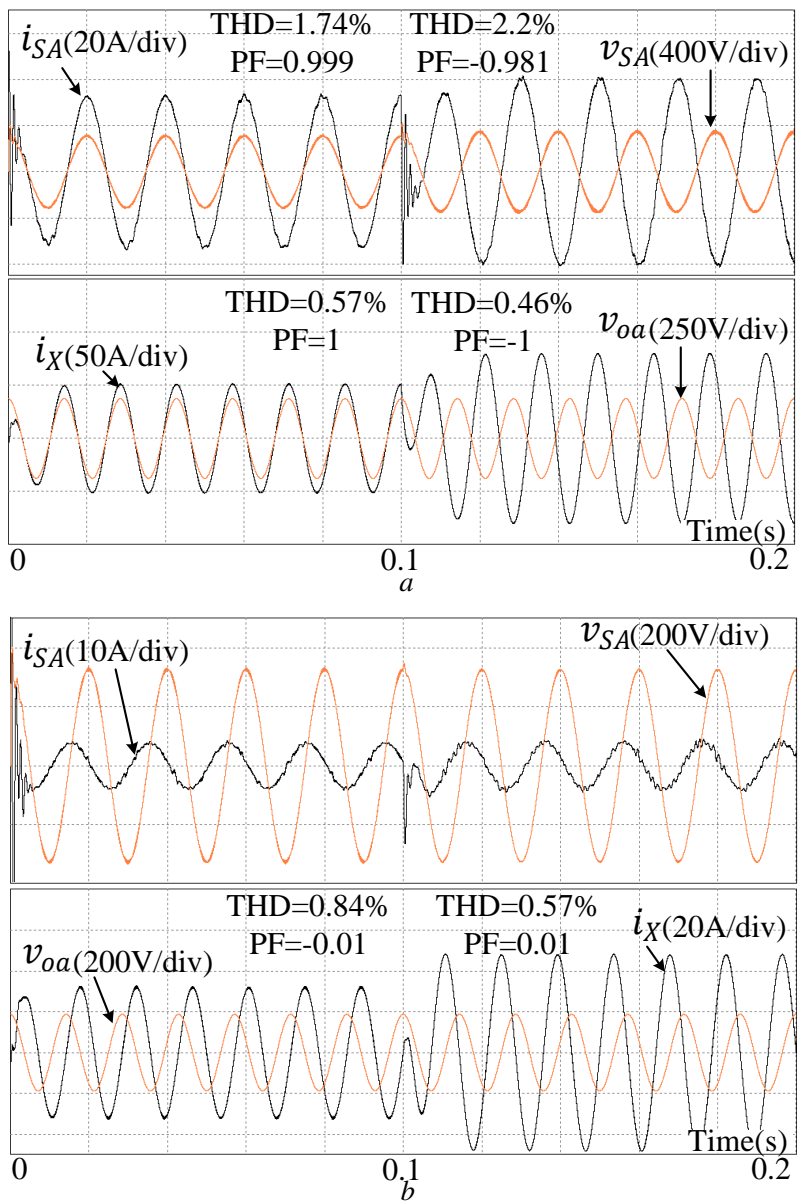

Fig. 16: The input source current and output current for bidirectional power-flow control, using a combination of damping resistor and digital filter with $R_{d}=10 \Omega$ and $\tau=0.2 \mathrm{~ms}$,

(a) Bidirectional active power-flow control, (b) Bidirectional reactive power-flow control

case of the damping resistor only, the converter efficiency reduces between $0.4 \%$ to $1.3 \%$, and the combination method between $0.1 \%$ to $0.4 \%$ for the output power range of $10 \%$ to $100 \%$.

\section{Comparison and Discussion}

The damping resistor and digital filter stabilization methods have advantages and disadvantages which can be optimized by the combination strategy. In the case of using only the digital filter, to filter out the input voltage, the main drawbacks are the power-up at the starting time, and the overvoltage at the transient times. These oscillations are eliminated in the damping resistor method. On the other hand, the extra power loss imposed by the damping resistors is eliminated in the digital filter strategy. Using the combination method leads to the same stability performance with larger damping resistances and less power loss. Furthermore, the high-frequency harmonics of the input current that can flow through the small damping resistor to the input source are reduced considerably. On the other hand, the existence of the damping resistor mitigates the power-up oscillations, and as there is no need for large values of $\tau$, the highfrequency disturbances of the input voltage cannot be reflected on the output side. The simulation results presented in section 9 are used to compare the performance of the three stabilization techniques. The comparison results are briefly presented in Table 2 .

There are some other factors effective on the system stability which cannot be included in the stabilization analysis using the small-signal modeling. Some of the main factors are the switching frequency, the switching period delay, the converter power losses, 
DSO-X 2004A, Mr57232158: Fri Jul 06 16:20:41 2018

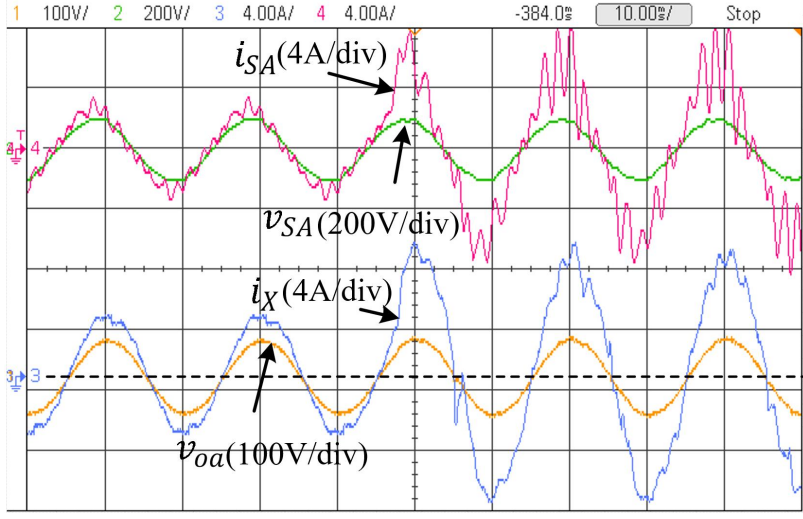

DSO-X 2004A, MY57232158: Fri Jul 06 15:54:55 2010

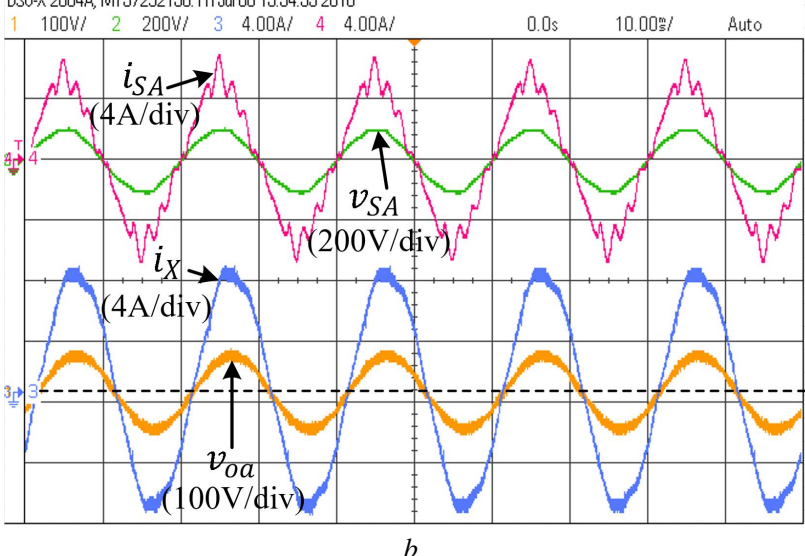

Fig. 17: Experimental results for bidirectional active power-flow control, input and output currents and voltages,

(a) Without applying any stabilization method (b) By adding only the large damping resistor $R_{d}=47 \Omega$

Table 2 Comparison of the MC performance using the proposed stabilization Methods

\begin{tabular}{|c|c|c|c|}
\hline & $\begin{array}{l}\text { Damping } \\
\text { resistor }\end{array}$ & $\begin{array}{l}\text { Digital } \\
\text { filter }\end{array}$ & $\begin{array}{l}\text { Combination } \\
\text { method }\end{array}$ \\
\hline Input current THD ${ }^{1}$ & $2.05 \%$ & $2.96 \%$ & $1.74 \%$ \\
\hline Output current THD ${ }^{1}$ & $0.55 \%$ & $0.62 \%$ & $0.57 \%$ \\
\hline Power loss & High & Zero & Low \\
\hline Transient oscillation & Negligible & High & Low \\
\hline overshoot & Very Low & Very high & Low \\
\hline Complexity & Low & Moderate & Moderate \\
\hline
\end{tabular}

${ }^{1}(q=0.82)$

the digital implementation of the modulation process, the commutation process of switching devices, and the number of branch switch overs (BSOs).

As presented in [16], a zero-order-hold can represent the analog to digital converter of the digital processor used for the modulation process which affects the stability. Also, an infinite switching frequency is assumed for the stability analysis, while the finite switching period causes one switching period delay $\left(T_{s}\right)$ related to the digital implementation of the control $[14,16]$.

The other impressive item is the turn-on and turn-off durations of the switching devices for the commutation process. The clock pulse frequency of the (FPGA) that is commonly used to control the commutation process needs to be adjusted properly according to one commutation step. By using the fast switches, it is possible to increase the FPGA clock pulse that is represented by the timestep in the simulation [29]. The designed DMC for this purpose implies a semisoft four-step current commutation. Considering the IGBT switching time limits, we select the FPGA clock frequency
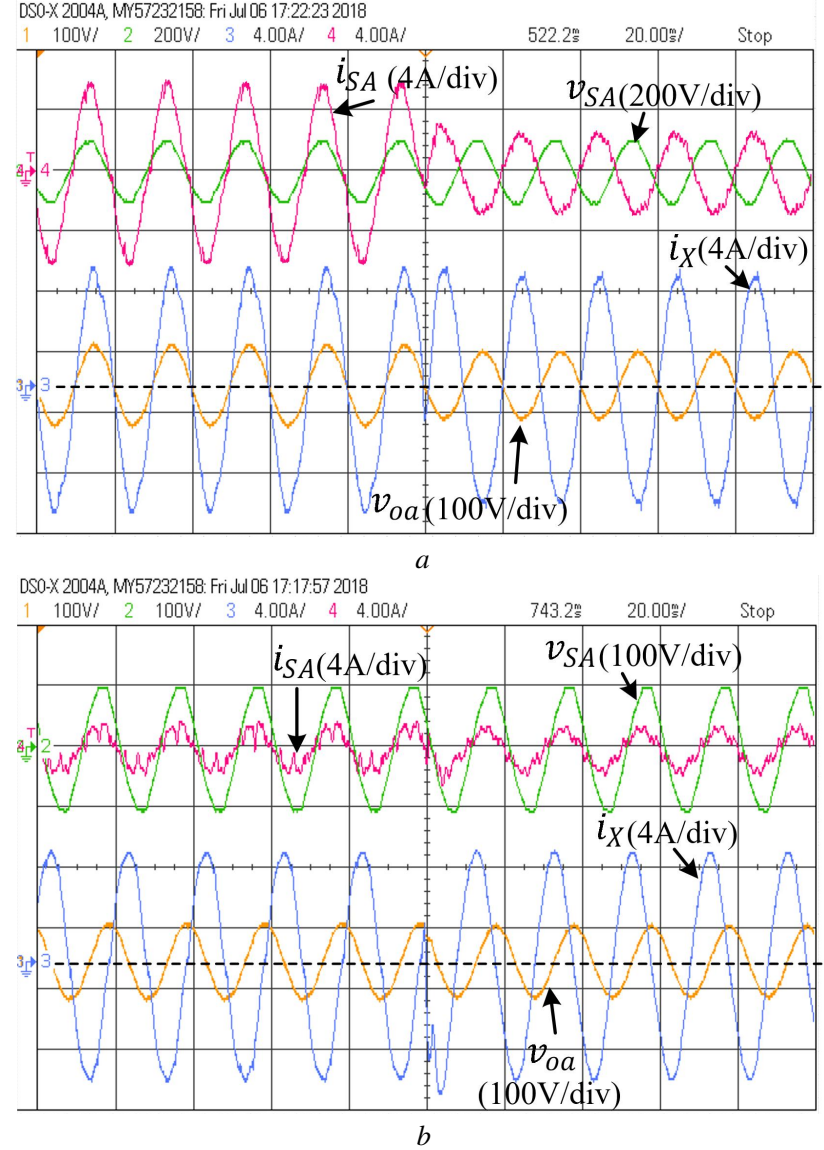

Fig. 18: Experimental results for bidirectional power-flow control, input and output currents and voltages, using the combination method, with $R_{d}=47 \Omega$ and $\tau=0.2 \mathrm{~ms}$,

(a) Bidirectional active power-flow control, (b) Bidirectional reactive power-flow control

as $25 \mathrm{MHz}$, or a time-step of $4 \mu s$ for simulation. It can be observed that reducing the time-step of the simulated circuit not only improves the THDs of the currents but also, improves the system stability for maximum voltage transfer ratio.

The total number of the state changes in a switching period can also affect the system stability. The number of BSOs for each output branches of the DMC in a switching period is determined by the switching pattern and the number of zero vectors utilized in the switching sequence in a cycle period. In the case of SVM with one zero vector, selecting a switching pattern with a double-sided vector sequence results in nine commutation steps and consequently eight BSOs for each switching period. This number increases to twelve BSOs when all the three zero vectors are added [30]. In order to reduce the output current THD, the number of zero vectors can be increased. However, since the input current THD improvement is not considerable, it causes more switching loss [31]. Using the anti-windup PI controllers instead of the normal PI controllers, and adjusting the limitations properly helps the stabilization in high voltage gains. This improvement is more evident in the experimental tests.

\section{Conclusion}

The stability analysis of the matrix converter system using the damping resistor and digital filter has been presented using the smallsignal model of the system, and a combination of both methods has been suggested in this paper. The effects of system parameters including the input filter, load, input and output source frequencies, and the line impedance on the system stability with and without using the stabilization techniques have been investigated. The 
DSO-X 2004A, MY57232158: Fri Jul 06 16:31:232018

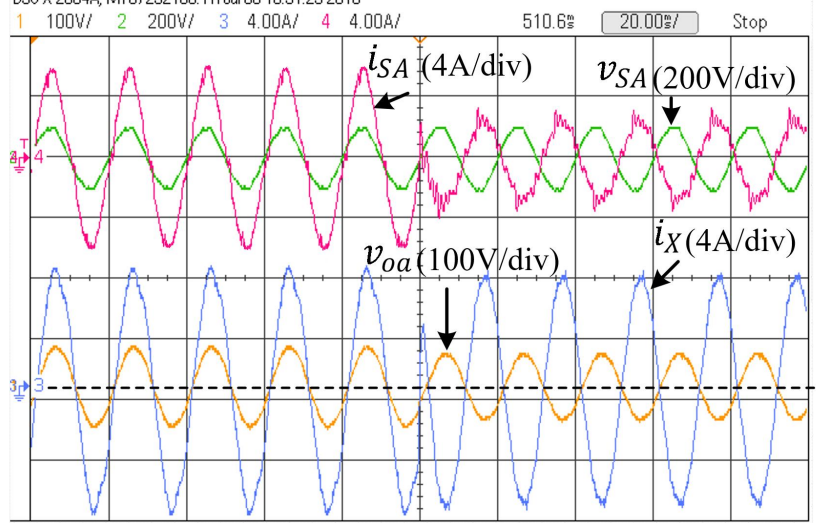

DSO-X 2004A, MY57232158: Fri Jul 06 16:40:492018

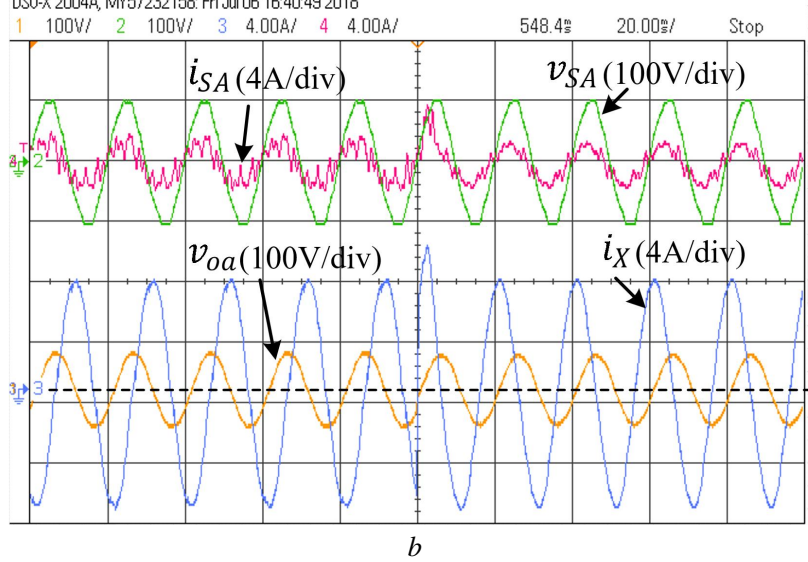

Fig. 19: Experimental results for bidirectional power-flow control, input and output currents and voltages, using the digital filter method, with $\tau=0.5 \mathrm{~ms}$,

(a) Bidirectional active power-flow control, (b) Bidirectional reactive power-flow control

performance of the methods in the stabilization of the MC for controlling bidirectional active and reactive power flows between two AC sources representing the utility grid and a microgrid was compared. The methods were compared regarding their input and output current THDs, transients, resultant power loss, and the complexity. It was shown that the suggested combination method presents better performance in transient behavior particularly in the case of connecting a low impedance supply such as a microgrid to the converter output. Besides, the system efficiency improved considerably using a larger damping resistance. In addition, the effects of the FPGA clock frequency, the switching pattern and anti-windup PI controller on the system stability were explained.

\section{References}

1 Rodriguez, J., Rivera, M., Kolar, J.W., Wheeler, P.W.: 'A review of control and modulation methods for matrix converters', IEEE Transactions on Industrial Electronics, 2012, 59, (1), pp. 58-70

2 Wheeler, P.W., Rodriguez, J., Clare, J.C., Empringham, L., Weinstein, A.: 'Matrix converters: a technology review', IEEE Transactions on Industrial Electronics, 2002, 49, (2), pp. 276-288

3 Barakati, S.M., Kazerani, M., Chen, X. 'A new wind turbine generation system based on matrix converter'. In: IEEE Power Engineering Society General Meeting. (San Francisco, CA, USA, 2005. pp. 2083-2089 Vol. 3

4 Rajendran, S., Govindarajan, U., Sankar, D.S.P.: 'Active and reactive power regulation in grid connected wind energy systems with permanent magnet synchronous generator and matrix converter', IET Power Electronics, 2014, 7, (3), pp. 591-603

5 Cardenas, R., Pena, R., Wheeler, P., Clare, J., Asher, G.: 'Control of the reactive power supplied by a wecs based on an induction generator fed by a matrix converter', IEEE Transactions on Industrial Electronics, 2009, 56, (2), pp. 429-438

6 Emadi, A., Khaligh, A., Rivetta, C.H., Williamson, G.A.: 'Constant power loads and negative impedance instability in automotive systems: definition, modeling, stability, and control of power electronic converters and motor drives', IEEE Transactions on Vehicular Technology, 2006, 55, (4), pp. 1112-1125
DSO-X 2004\&, M557232158. Fri Jul 06 16:57:46 2018
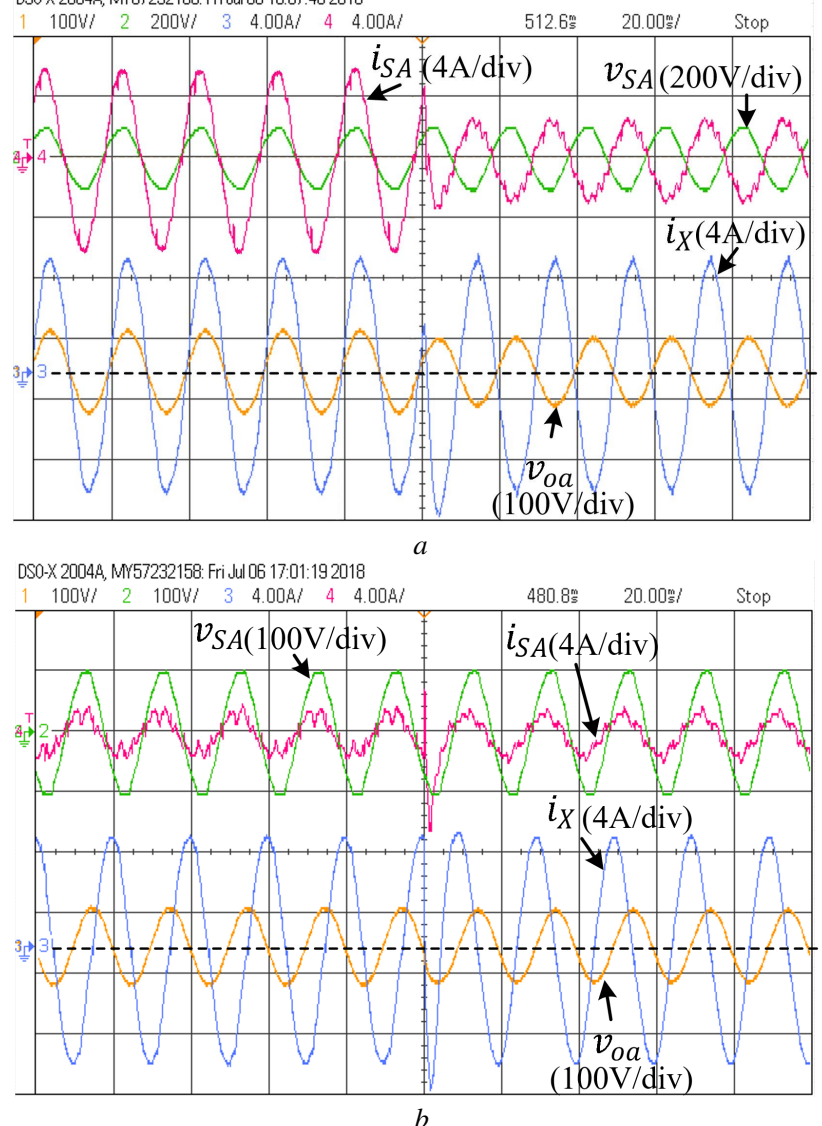

Fig. 20: Experimental results for bidirectional power-flow control, input and output currents and voltages, using the damping resistor method, with $R_{d}=3 \Omega$,

(a) Bidirectional active power-flow control, (b) Bidirectional reactive power-flow control

7 Rahimi, A.M., Emadi, A.: 'Active damping in dc/dc power electronic converters: A novel method to overcome the problems of constant power loads', IEEE Transactions on Industrial Electronics, 2009, 56, (5), pp. 1428-1439

8 Liu, X., Forsyth, A.J., Cross, A.M.: 'Negative input-resistance compensator for a constant power load', IEEE Transactions on Industrial Electronics, 2007, 54, (6), pp. 3188-3196

9 Chandrasekaran, S., Borojevic, D., Lindner, D.K. 'Input filter interaction in three phase ac-dc converters'. In: 30th Annual IEEE Power Electronics Specialists Conference. Record. (Cat. No.99CH36321). vol. 2. (Charleston, SC, USA, USA, 1999. pp. 987-992 vol.2

10 Casadei, D., Serra, G., Tani, A., Zarri, L. 'Stability analysis of electrical drives fed by matrix converters'. In: Industrial Electronics, 2002. ISIE 2002. Proceedings of the 2002 IEEE International Symposium on. vol. 4. (L'Aquila, Italy, 2002. pp. $1108-1113$ vol. 4

11 Singer, S.: 'Realization of loss-free resistive elements', IEEE Transactions on Circuits and Systems, 1990, 37, (1), pp. 54-60

12 Singer, S., Ozeri, S., Shmilovitz, D.: 'A pure realization of loss-free resistor', IEEE Transactions on Circuits and Systems I: Regular Papers, 2004, 51, (8), pp. 1639 1647

13 Casadei, D., Serra, G., Tani, A., Trentin, A., Zarri, L.: 'Theoretical and experimental investigation on the stability of matrix converters', IEEE Transactions on Industrial Electronics, 2005, 52, (5), pp. 1409-1419

14 Casadei, D., Serra, G., Tani, A., Zarri, L.: 'Effects of input voltage measurement on stability of matrix converter drive system', IEE Proceedings - Electric Power Applications, 2004, 151, (4), pp. 487-497

15 Liu, F., Klumpner, C., Blaabjerg, F. 'Stability analysis and experimental evaluation of a matrix converter drive system'. In: Industrial Electronics Society, 2003. IECON '03. The 29th Annual Conference of the IEEE. vol. 3. (Roanoke, VA, USA, USA, 2003. pp. 2059-2065 Vol.3

16 Ruse, C.A.J., Clare, J.C., Klumpner, C. 'Numerical approach for guaranteeing stable design of practical matrix converter drive systems'. In: IECON 2006 32nd Annual Conference on IEEE Industrial Electronics. (Paris, France, 2006. pp. 2630-2635

17 Sun, Y., Su, M., Li, X., Wang, H., Gui, W.: 'A general constructive approach to matrix converter stabilization', IEEE Transactions on Power Electronics, 2013, 28, (1), pp. 418-431

18 Klumpner, C., Blaabjerg, F. chapter 3. In: 'Fundamentals of the matrix converter technology'. (United States: Academic Press, 2002. 
19 Rodriguez, J., Silva, E., Blaabjerg, F., Wheeler, P., Clare, J., Pontt, J.: 'Matrix converter controlled with the direct transfer function approach: Analysis, modelling and simulation', International Journal of Electronics, 2005, 92, pp. 63-85

20 She, H., Lin, H., Wang, X., Yue, L. 'Damped input filter design of matrix converter'. In: 2009 International Conference on Power Electronics and Drive Systems (PEDS). (Taipei, Taiwan, 2009. pp. 672-677

21 Xiao, D., Rahman, M.F.: 'Sensorless direct torque and flux controlled ipm synchronous machine fed by matrix converter over a wide speed range', IEEE Transactions on Industrial Informatics, 2013, 9, (4), pp. 1855-1867

22 Kume, T., Yamada, K., Higuchi, T., Yamamoto, E., Hara, H., Sawa, T., et al.: 'Integrated filters and their combined effects in matrix converter', IEEE Transactions on Industry Applications, 2007, 43, (2), pp. 571-581

23 Gibbard, M.J., Pourbeik, P., Vowles, D.: 'Small-signal stability, control and dynamic performance of power systems'. (University of Adelaide Press Adelaide, South Australia, 2015)

24 Wheeler, P., Grant, D.: 'Optimised input filter design and low-loss switching techniques for a practical matrix converter', IEE Proceedings - Electric Power Applications, 1997, 144, (1), pp. 53-60

25 D. Casadei and G. Serra and A. Tani and L. Zarri: 'Matrix converter modulation strategies: a new general approach based on space-vector representation of the switch state', IEEE Transactions on Industrial Electronics, 2002, 49, (2), pp. 370-381

26 Casadei, D., Serra, G., Tani, A.: 'Reduction of the input current harmonic content in matrix converters under input/output unbalance', IEEE Transactions on Industrial Electronics, 1998, 45, (3), pp. 401-411

27 Schauder, C., Mehta, H.: 'Vector analysis and control of advanced static var compensators', IEE Proceedings C - Generation, Transmission and Distribution, 1993, 140, (4), pp. 299-306

28 Gao, F., Iravani, M.R.: 'Dynamic model of a space vector modulated matrix converter', IEEE Transactions on Power Delivery, 2007, 22, (3), pp. 1696-1705

29 Ricci, F., Le.Huy, H.: 'Modeling and simulation of fpga-based variable-speed drives using simulink', Mathematics and Computers in Simulation, 2003, 63, pp. 183-195

30 Nielsen, P., Blaabjerg, F., Pedersen, J.K. 'Space vector modulated matrix converter with minimized number of switchings and a feedforward compensation of input voltage unbalance'. In: Proceedings of International Conference on Power Electronics, Drives and Energy Systems for Industrial Growth. vol. 2. (New Delhi, India, India, 1996. pp. 833-839 vol.2

31 Malekjamshidi, Z., Jafari, M., Xiao, D., Zhu, J. 'Operation of indirect matrix converters in different svm switching patterns'. In: 2015 4th International Conference on Electric Power and Energy Conversion Systems (EPECS). (Sharjah, United Arab Emirates, 2015. pp. 1-5 